Logic and Logical Philosophy

Volume 18 (2009), 97-126

DOI: $10.12775 /$ LLP.2009.007

\title{
Norihiro Kamide
}

\section{TEMPORAL NON-COMMUTATIVE LOGIC: Expressing time, resource, order and hierarchy}

\begin{abstract}
A first-order temporal non-commutative logic $\mathrm{TN}[l]$, which has no structural rules and has some $l$-bounded linear-time temporal operators, is introduced as a Gentzen-type sequent calculus. The logic TN $[l]$ allows us to provide not only time-dependent, resource-sensitive, ordered, but also hierarchical reasoning. Decidability, cut-elimination and completeness (w.r.t. phase semantics) theorems are shown for $\mathrm{TN}[l]$. An advantage of $\mathrm{TN}[l]$ is its decidability, because the standard first-order linear-time temporal logic is undecidable. A correspondence theorem between $\mathrm{TN}[l]$ and a resourceindexed non-commutative logic $\mathrm{RN}[l]$ is also shown. This theorem is intended to state that "time" is regarded as a "resource".
\end{abstract}

Keywords: Temporal non-commutative logic, cut-elimination, sequent calculus, completeness, decidability.

\section{Introduction}

\subsection{Time}

Temporal logics are modal logics for describing the temporal ordering of events. Two possible views regarding the nature of time induce two types of temporal logics: linear-time temporal logic (LTL) and branching-time temporal logic. It is known that LTL is very useful for verifying and specifying concurrent systems. Gentzen-type sequent calculi for LTL and its neighbors have been introduced by many researchers. For example, a sequent calculus $\mathrm{LT}_{\omega}$ for Kröger's first-order infinitary LTL [14] was introduced by Kawai 
[12]. He proved the cut-elimination and completeness theorems for $\mathrm{LT}_{\omega}$. An alternative proof of the cut-elimination theorem for $\mathrm{LT}_{\omega}$ was given by Kamide [11] introducing an embedding of $\mathrm{LT}_{\omega}$ into a sequent calculus for infinitary logic.

In the present paper, a first-order, intuitionistic, non-commutative and bounded version of $\mathrm{LT}_{\omega}$, which has an embedding into an intuitionistic noncommutative logic FL (full Lambek logic) rather than infinitary logic, is studied. This logic is called a temporal non-commutative logic TN $[l]$. The cut-elimination, embedding (into FL), decidability and completeness (w.r.t. phase semantics) theorems for $\mathrm{TN}[l]$ are proved as the main results of this paper. In particular, the decidability is an advantage of $\mathrm{TN}[l]$, because the standard first-order LTL is undecidable. $\mathrm{TN}[l]$ is intended to obtain a useful theoretical basis for adequately representing not only time-dependent, resource-sensitive, ordered, but also hierarchical reasoning. In this paper, a resource-indexed non-commutative logic $\mathrm{RN}[l]$ is also introduced, and a correspondence theorem between $\mathrm{TN}[l]$ and $\mathrm{RN}[l]$ is shown. This theorem is intended to state that "time" is regarded as a "resource".

Although the standard LTL has an infinite (unbounded) time domain, i.e., the set $\omega$ of all natural numbers, the logic $\mathrm{TN}[l]$ has a bounded time domain which is restricted by a fixed positive integer $l$, i.e., the set $\omega_{l}:=\{x \in$ $\omega \mid x \leq l\}$. For the virtue of the bounded time domain, the embedding (into $\mathrm{FL}$ ), cut-elimination and decidability theorems for TN $[l]$ can be shown. Such theoretical merits may not be obtained for the unbounded version $\mathrm{TN}[\omega]$ of $\mathrm{TN}[l]$, because the unbounded time domain requires some infinite inference rules. Whereas $\mathrm{LT}_{\omega}$ informally characterizes the Hilbert-style axiom scheme for the temporal operators $\mathrm{G}$ (globally) and $\mathrm{X}$ (next):

$$
\mathrm{G} \alpha \leftrightarrow\left(\alpha \wedge \mathrm{X} \alpha \wedge \mathrm{X}^{2} \alpha \wedge \cdots \infty\right)
$$

where $\mathrm{X}^{i} \alpha$ means $\overbrace{\mathrm{XX} \cdots \mathrm{X}}^{i} \alpha$, the logic $\mathrm{TN}[l]$ characterizes the Hilbert-style axiom scheme:

$$
\mathrm{G} \alpha \leftrightarrow\left(\alpha \wedge \mathrm{X} \alpha \wedge \mathrm{X}^{2} \alpha \wedge \cdots \wedge \mathrm{X}^{l} \alpha\right),
$$

which is regarded as a finite approximation of the original one.

To restrict the time domain in LTL is not a new idea. Such an idea was discussed, for example, in $[3,4,6]$. It is known that to restrict the time domain is a technique that may be applied to obtain a decidable or efficient fragment of LTL [6]. Restricting the time domain implies not only some purely theoretical merits as mentioned above, but also some practical merits 
for describing temporal databases [4] and for implementing an efficient model checking algorithm, called bounded model checking [3]. Such practical merits are important due to the fact that there are problems in computer science and artificial intelligence where only a finite fragment of the time sequence is of interest [4].

\subsection{Resource}

The notion of "resource", encompassing concepts such as processor time, memory, cost of components and energy requirements, is fundamental to computational systems [17]. This notion is also very important in handling real scheduling problems to construct complex plans of actions, since many actions consume resources, such as money, gas and raw materials [18]. "Time" is also regarded as a "resource". This may be justified in that the concept of "time" in computer systems, such as CPU-time in process scheduling, is considered to be a "resource". Similarly, in the real world, "time is money, i.e., resources". This "time-as-resources" interpretation will also be justified by the corresponding theorem between $\mathrm{TN}[l]$ and $\mathrm{RN}[l]$.

An approach towards a logical theory of resources has been developed by Pym, O'Hearn and Yang [17], using the logic BI of bunched implications, which is an extension of a linear logic. It is known that Girard's linear logics [5], i.e., logics that have no contraction rule:

$$
\frac{\Gamma, \alpha, \alpha, \Delta \Rightarrow \gamma}{\Gamma, \alpha, \Delta \Rightarrow \gamma}
$$

can elegantly represent the concept of "resource consumption". An appropriate resource consumption example is medicine consumption in medical reasoning. Consider a medicine $m$ as a resource. An expression $m(x) \Rightarrow$ recover $(x)$ means "if a person $x$ uses a medicine $m x$ makes a recovery from the disease with the medicine". In this case, $m(x), m(x) \Rightarrow \operatorname{recover}(x)$ and $m(x) \Rightarrow$ recover $(x)$ have the completely different meaning, because two medicines and one medicine have the different effect in general. If assuming the classical or intuitionistic logic, then these two sequents are logically equivalent because of the presence of the contraction rule.

Due to the usefulness of combining linear logic with temporal logic, a number of temporal linear logics have been introduced and studied by many researchers (see e.g., [7] and the references therein). On the other hand, a temporal non-commutative logic, which is based on Lambek calculus [15], has not been proposed yet. Such a temporal non-commutative logic is useful 
for expressing not only time-dependent, resource-sensitive, ordered, but also hierarchical reasoning, i.e., more fine-grained resource-sensitive reasoning can be expressed using such a logic. This is the reason why we introduce $\mathrm{TN}[l]$.

\subsection{Order}

Non-commutative logics are substructural logics without the exchange rule:

$$
\frac{\Gamma, \beta, \alpha, \Delta \Rightarrow \gamma}{\Gamma, \alpha, \beta, \Delta \Rightarrow \gamma}
$$

Although a number of non-commutative logics have been proposed and studied by many researchers, the original and basic non-commutative logic is Lambek calculus [15]. An enrichment FL of the Lambek calculus by full set of connectives has also been studied by many logicians. The proposed logic $\mathrm{TN}[l]$ is an extension of FL.

In the case of medicine consumption discussed before, it may not be sufficient to consider the effects of medicines. For example, if we consider two distinct medicines $m_{1}$ and $m_{2}$, then the meanings of the following two expressions are regarded as different: $m_{1}(x), m_{2}(x) \Rightarrow \operatorname{recover}(x)$ and $m_{2}(x), m_{1}(x) \Rightarrow$ recover $(x)$, because the order of using medicines change the effect of the medicines. In other words, the order or priority of using medicines is more important in general. A more detailed example is expressed below. An expression meal $(x)$ means "a person $x$ has a meal". Then, $m(x)$, meal $(x) \Rightarrow$ recover $(x)$ and meal $(x), m(x) \Rightarrow$ recover $(x)$ have the different meaning, i.e., the effect of the medicine $m$ is different whether the medicine is used after or before the meal.

To express such fine-grained resource-sensitive reasoning, we have to use a non-commutative logic such as FL, because, for example, logics with the exchange rule cannot express the order of descending priorities of the use of medicines. It can be understood that in a sequent expression $\gamma_{1}, \gamma_{2}, \ldots, \gamma_{n} \Rightarrow$ $\beta$ in FL, the antecedent $\left(\gamma_{1}, \gamma_{2}, \ldots, \gamma_{n}\right)$ can express the order or priority of consuming the resources $\gamma_{1}, \gamma_{2}, \ldots, \gamma_{n}$, indeed, $\left(\gamma_{1}, \gamma_{2}, \ldots, \gamma_{n}\right)$ is a sequence of formulas in FL, since it has no exchange rule. Remark that two sequents $\gamma_{1}, \gamma_{2}, \ldots, \gamma_{n} \Rightarrow \beta$ and $\gamma_{1} * \gamma_{2} * \cdots * \gamma_{n} \Rightarrow \beta$ where $*$ is the fusion connective are logically equivalent in FL, and hence an expression $\gamma_{1} * \gamma_{2}$ means "first $\gamma_{1}$ is consumed, next so is $\gamma_{2}$ ". Also remark that in two expressions $\alpha \rightarrow \beta$ and $\alpha \leftarrow \beta$, the implications $\rightarrow$ and $\leftarrow$ represent resource consumption with 
priority, e.g., $\rightarrow$ means the consumption of (subscription) descending order priority, and $\leftarrow$ means the consumption of ascending order priority.

The following realistic time-dependent expression, which represents a liveness property, can be obtained in $\mathrm{TN}[l]$ by using the $l$-bounded temporal globally and eventually operators $\mathrm{G}$ and $\mathrm{F}$, respectively:

$$
\mathrm{G}(\operatorname{meal}(x) * m(x) \rightarrow \mathrm{F} \text { recover }(x))
$$

which means "if a person $x$ eats a meal and takes a medicine $m$ in this order, then $x$ will eventually make a recovery from the disease".

\subsection{Hierarchy}

In FL, a preference statement: "I prefer $\alpha$ to $\beta$ " can be expressed formally as $\alpha, \beta \Rightarrow \gamma$ where $\gamma$ indicates a common sort of $\alpha$ and $\beta$. A preference expression: $\alpha_{1}, \alpha_{2}, \alpha_{3}, \ldots, \alpha_{n} \Rightarrow \gamma$ means a descending order of preferences. Examples of this expression, which were presented in [9], are:

$$
\begin{aligned}
& \operatorname{fruit}(x), \operatorname{vegetable}(x) \Rightarrow \operatorname{food}(x) \\
& \operatorname{apple}(x), \operatorname{orange}(x), \operatorname{banana}(x) \Rightarrow \operatorname{fruit}(x) \\
& \operatorname{tomato}(x), \operatorname{carrot}(x), \operatorname{cucumber}(x) \Rightarrow \operatorname{vegetable}(x) \\
& \text { Taiwan-banana }(x), \text { Philippine-banana }(x) \Rightarrow \operatorname{banana}(x)
\end{aligned}
$$

which represent the preference of a person $x$ for foods, and also address the following tree that represents a taxonomic hierarchy for foods:

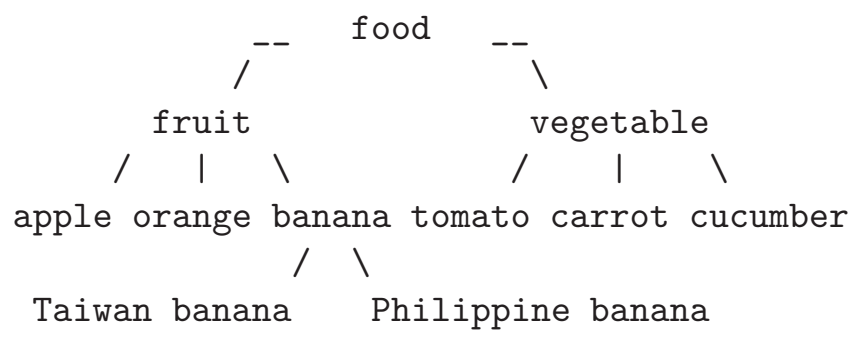

A sequent calculus-based proof representation of this taxonomic tree is:

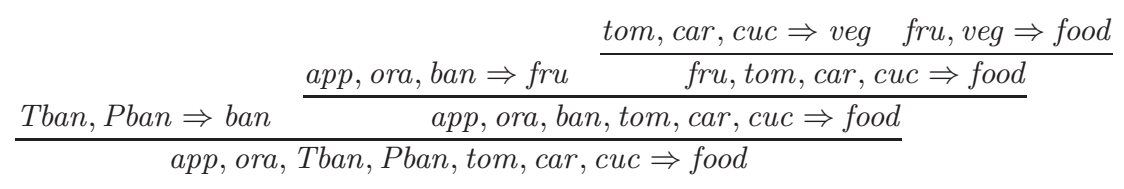

where the names of the foods are abbreviated. 
Tree expressions for hierarchies are known as very important for describing some programming languages such as XML (extensible markup language). Indeed, XML documents are sometimes expressed by a kind of labeled trees. By using TN $[l]$, more expressive and informative hierarchies can be presented. For example, some timed or labeled hierarchies may be expressed in $\mathrm{TN}[l]$ by using the $l$-bounded temporal next-time operator $\mathrm{X}$.

\subsection{Summary of this paper}

The contents of this paper are then summarized as follows.

In Section 2, the logic TN $[l]$ is introduced as an extension of FL with the addition of some $l$-bounded temporal operators, and the embedding theorem of $\mathrm{TN}[l]$ into FL is shown. The cut-elimination and decidability theorems for $\mathrm{TN}[l]$ are obtained as a consequence of the embedding theorem.

In Section 3, a phase semantics for the propositional fragment of $\mathrm{TN}[l]$ is introduced, and the completeness theorem with respect to this semantics is proved. As a consequence of this theorem, an alternative (semantic) proof of the cut-elimination theorem for $\mathrm{TN}[l]$ is also obtained. Although the completeness theorem for the first-order version can be shown, such a discussion is omitted here, since the essential temporal part of this semantics is completely included in the propositional part.

In Section 4, the logic RN $[l]$ is introduced as an extension of FL with the addition of some $l$-bounded exponential operators, and the correspondence theorem between $\mathrm{RN}[l]$ and $\mathrm{TN}[l]$ is shown. As a consequence of this theorem, an alternative (embedding-based) proof of the cut-elimination theorems for $\mathrm{TN}[l]$ and $\mathrm{RN}[l]$ is also obtained.

In Section 5, this paper is concluded, and some substructural extensions of the present paper's results are discussed.

\section{Temporal non-commutative logic}

The following list of symbols is used for the language of the underlying logics: free variables $a_{0}, a_{1}, \ldots$, bound variables $x_{0}, x_{1}, \ldots$, functions $f_{0}, f_{1}, \ldots$, predicates $p_{0}, p_{1}, \ldots, \mathbf{1}$ (multiplicative truth constant), $\top$ (additive truth constant), $\perp$ (additive falsity constant), $\rightarrow$ (right implication), $\leftarrow$ (left implication), $\wedge$ (conjunction), $\vee$ (disjunction), $*$ (fusion), $\forall$ (any), $\exists$ (exists), X (next), G (globally) and F (eventually). The numbers of free and bound variables are assumed to be countable, and the numbers of functions and predicates are also assumed to be countable. It is also assumed that there 
is at least one predicate. A 0 -ary function is an individual constant, and a 0 -ary predicate is a propositional variable.

Greek lower-case letters $\alpha, \beta, \ldots$ are used for formulas, and Greek capital letters $\Gamma, \Delta, \ldots$ are used for finite (possibly empty) sequences of formulas. For any $\sharp \in\{X, G, F\}$, an expression $\sharp \Gamma$ is used to denote the sequence $\langle\sharp \gamma \mid \gamma \in \Gamma\rangle$. The symbol $\equiv$ is used to denote the equality of sequences of symbols. The symbol $\omega$ is used to represent the set of natural numbers. Let $l$ be a fixed positive integer. Then, the symbol $\omega_{l}$ is used to represent the set $\{i \in \omega \mid i \leq l\}$. An expression $\mathrm{X}^{i} \alpha$ for any $i \in \omega$ is defined inductively by $\left(\mathrm{X}^{0} \alpha \equiv \alpha\right)$ and $\left(\mathrm{X}^{n+1} \alpha \equiv \mathrm{XX}^{n} \alpha\right)$. Lower-case letters $i$ and $j$ are used to denote any natural numbers.

An expression of the form $\Gamma \Rightarrow \gamma$ where $\gamma$ is a single formula is called a sequent. It is assumed that the terminological conventions regarding sequent calculus (e.g., antecedent, succedent etc.) are the usual ones. If a sequent $S$ is provable in a sequent calculus $L$, then such a fact is denoted as $L \vdash S$ or $\vdash S$. The parentheses for $*$ are omitted since $*$ is associative, i.e., $\vdash$ $\alpha *(\beta * \gamma) \Rightarrow(\alpha * \beta) * \gamma$ and $\vdash(\alpha * \beta) * \gamma \Rightarrow \alpha *(\beta * \gamma)$ for any formulas $\alpha$, $\beta$ and $\gamma$.

A temporal non-commutative logic $\mathrm{TN}[l]$ is introduced below.

Definition 2.1 (TN[l]). Let $l$ be a fixed positive integer (called a time bound). The initial sequents of $\mathrm{TN}[l]$ are of the form: for any atomic formula $p$,

$$
\mathrm{X}^{i} p \Rightarrow \mathrm{X}^{i} p \quad \Rightarrow \mathrm{X}^{i} \mathbf{1} \quad \Gamma, \mathrm{X}^{i} \perp, \Delta \Rightarrow \gamma \quad \Gamma \Rightarrow \mathrm{X}^{i} \top .
$$

The cut rule of $\mathrm{TN}[l]$ is of the form:

$$
\frac{\Gamma \Rightarrow \alpha \quad \Sigma, \alpha, \Delta \Rightarrow \gamma}{\Sigma, \Gamma, \Delta \Rightarrow \gamma} \text { (cut) }
$$

The logical inference rules of $\mathrm{TN}[l]$ are of the form: for any $k \in \omega_{l}$ and any positive integer $m$,

$$
\begin{array}{cl}
\frac{\Gamma, \Delta \Rightarrow \gamma}{\Gamma, \mathrm{X}^{i} \mathbf{1}, \Delta \Rightarrow \gamma}(\mathbf{1 w e}) \\
\frac{\Gamma \Rightarrow \mathrm{X}^{i} \alpha \quad \Sigma, \mathrm{X}^{i} \beta, \Delta \Rightarrow \gamma}{\Sigma, \mathrm{X}^{i}(\alpha \rightarrow \beta), \Gamma, \Delta \Rightarrow \gamma}(\rightarrow \text { left }) & \frac{\Gamma, \mathrm{X}^{i} \alpha \Rightarrow \mathrm{X}^{i} \beta}{\Gamma \Rightarrow \mathrm{X}^{i}(\alpha \rightarrow \beta)}(\rightarrow \text { right }) \\
\frac{\Gamma \Rightarrow \mathrm{X}^{i} \alpha \quad \Sigma, \mathrm{X}^{i} \beta, \Delta \Rightarrow \gamma}{\Sigma, \Gamma, \mathrm{X}^{i}(\alpha \leftarrow \beta), \Delta \Rightarrow \gamma}(\leftarrow \text { left }) & \frac{\mathrm{X}^{i} \alpha, \Gamma \Rightarrow \mathrm{X}^{i} \beta}{\Gamma \Rightarrow \mathrm{X}^{i}(\alpha \leftarrow \beta)}(\leftarrow \text { right })
\end{array}
$$




$$
\begin{aligned}
& \frac{\Gamma, \mathrm{X}^{i} \alpha, \Delta \Rightarrow \gamma}{\Gamma, \mathrm{X}^{i}(\alpha \wedge \beta), \Delta \Rightarrow \gamma}(\wedge \operatorname{left} 1) \frac{\Gamma, \mathrm{X}^{i} \beta, \Delta \Rightarrow \gamma}{\Gamma, \mathrm{X}^{i}(\alpha \wedge \beta), \Delta \Rightarrow \gamma}(\wedge \text { left2 }) \\
& \frac{\Gamma \Rightarrow \mathrm{X}^{i} \alpha \quad \Gamma \Rightarrow \mathrm{X}^{i} \beta}{\Gamma \Rightarrow \mathrm{X}^{i}(\alpha \wedge \beta)}(\text { right }) \quad \frac{\Gamma, \mathrm{X}^{i} \alpha, \Delta \Rightarrow \gamma \quad \Gamma, \mathrm{X}^{i} \beta, \Delta \Rightarrow \gamma}{\Gamma, \mathrm{X}^{i}(\alpha \vee \beta), \Delta \Rightarrow \gamma} \text { (Vleft) } \\
& \frac{\Gamma \Rightarrow \mathrm{X}^{i} \alpha}{\Gamma \Rightarrow \mathrm{X}^{i}(\alpha \vee \beta)}\left(\text { Vright1) } \frac{\Gamma \Rightarrow \mathrm{X}^{i} \beta}{\Gamma \Rightarrow \mathrm{X}^{i}(\alpha \vee \beta)}(\text { Vright2) }\right. \\
& \frac{\Gamma, \mathrm{X}^{i} \alpha, \mathrm{X}^{i} \beta, \Delta \Rightarrow \gamma}{\Gamma, \mathrm{X}^{i}(\alpha * \beta), \Delta \Rightarrow \gamma}(* \text { left }) \quad \frac{\Gamma \Rightarrow \mathrm{X}^{i} \alpha \quad \Delta \Rightarrow \mathrm{X}^{i} \beta}{\Gamma, \Delta \Rightarrow \mathrm{X}^{i}(\alpha * \beta)}(* \text { right }) \\
& \left.\frac{\Gamma, \mathrm{X}^{i} \alpha(t), \Delta \Rightarrow \gamma}{\Gamma, \mathrm{X}^{i} \forall x \alpha(x), \Delta \Rightarrow \gamma}(\forall \text { left }) \quad \frac{\Gamma \Rightarrow \mathrm{X}^{i} \alpha(a)}{\Gamma \Rightarrow \mathrm{X}^{i} \forall x \alpha(x)} \text { ( } \forall \text { right }\right) \\
& \frac{\Gamma, \mathrm{X}^{i} \alpha(a), \Delta \Rightarrow \gamma}{\Gamma, \mathrm{X}^{i} \exists x \alpha(x), \Delta \Rightarrow \gamma}(\exists \text { left }) \quad \frac{\Gamma \Rightarrow \mathrm{X}^{i} \alpha(t)}{\Gamma \Rightarrow \mathrm{X}^{i} \exists x \alpha(x)}(\exists \text { right }) \\
& \frac{\Gamma, \mathrm{X}^{l} \alpha, \Delta \Rightarrow \gamma}{\Gamma, \mathrm{X}^{l+m} \alpha, \Delta \Rightarrow \gamma} \text { (Xleft) } \frac{\Gamma \Rightarrow \mathrm{X}^{l} \alpha}{\Gamma \Rightarrow \mathrm{X}^{l+m} \alpha} \text { (Xright) } \\
& \frac{\Gamma, \mathrm{X}^{i+k} \alpha, \Delta \Rightarrow \gamma}{\Gamma, \mathrm{X}^{i} \mathrm{G} \alpha, \Delta \Rightarrow \gamma} \text { (Gleft) } \frac{\left\{\Gamma \Rightarrow \mathrm{X}^{i+j} \alpha\right\}_{j \in \omega_{l}}}{\Gamma \Rightarrow \mathrm{X}^{i} \mathrm{G} \alpha} \text { (Gright) } \\
& \frac{\left\{\Gamma, \mathrm{X}^{i+j} \alpha, \Delta \Rightarrow \gamma\right\}_{j \in \omega_{l}}}{\Gamma, \mathrm{X}^{i} \mathrm{~F} \alpha, \Delta \Rightarrow \gamma} \text { (Fleft) } \quad \frac{\Gamma \Rightarrow \mathrm{X}^{i+k} \alpha}{\Gamma \Rightarrow \mathrm{X}^{i} \mathrm{~F} \alpha} \text { (Fright) }
\end{aligned}
$$

where $a$ in ( $\forall$ right) and ( $\exists$ left) is a free variable which must not occur in the lower sequents of the rules, and $t$ in ( $\forall$ left) and ( $\exists$ right) is an arbitrary term.

Definition $2.2(\mathrm{TN}[\omega])$. The logic $\mathrm{TN}[\omega]$ is obtained from $\mathrm{TN}[l]$ by deleting $\{($ Xleft $)$, (Xright) $\}$ and replacing $\omega_{l}$ by $\omega$. The modified rules obtained from (Gleft), (Gright), (Fleft) and (Fright) are denoted as (Gleft $\left.{ }^{\omega}\right)$, $\left(\right.$ Gright $\left.^{\omega}\right)$, $\left(\right.$ Fleft $\left.^{\omega}\right)$ and $\left(\right.$ Fright $\left.^{\omega}\right)$, respectively.

Definition 2.3 (FL). The logic FL (full Lambek logic) is obtained from $\mathrm{TN}[l]$ by deleting (Xleft), (Xright), (Gleft), (Gright), (Fleft), (Fright) and replacing $\mathrm{X}^{i}$ by $\mathrm{X}^{0}$ (i.e., all the occurrences of $\mathrm{X}$ in the inference rules are deleted). The modified inference rules for FL by replacing $i$ by 0 are denoted by labeling "FL" in superscript position, e.g., $\left(\rightarrow \operatorname{left}^{F L}\right)$. 
Note that (Gright) and (Fleft) have $l+1$ (i.e., finite number of) premises, e.g., in the case $l=3$, (Gright) has four premises:

$$
\frac{\Gamma \Rightarrow \mathrm{X}^{i} \alpha \quad \Gamma \Rightarrow \mathrm{X}^{i+1} \alpha \quad \Gamma \Rightarrow \mathrm{X}^{i+2} \alpha \quad \Gamma \Rightarrow \mathrm{X}^{i+3} \alpha}{\Gamma \Rightarrow \mathrm{X}^{i} \mathrm{G} \alpha} \text { (Gright). }
$$

In (Gleft) and (Fright), the number $k$ is bounded by $l$. Then, TN $[l]$ has the Hilbert-style axiom schemes:

$$
\begin{aligned}
& \mathrm{G} \alpha \leftrightarrow\left(\alpha \wedge \mathrm{X} \alpha \wedge \mathrm{X}^{2} \alpha \wedge \cdots \wedge \mathrm{X}^{l} \alpha\right), \\
& \mathrm{F} \alpha \leftrightarrow\left(\alpha \vee \mathrm{X} \alpha \vee \mathrm{X}^{2} \alpha \vee \cdots \vee \mathrm{X}^{l} \alpha\right) .
\end{aligned}
$$

By (Xleft) and (Xright), the nest of the outermost occurrence of $\mathrm{X}$ in a formula can be bounded by $l$. Indeed, (Xleft) and (Xright) correspond to the Hilbert-style axiom scheme

$$
\mathrm{X}^{l+m} \alpha \leftrightarrow \mathrm{X}^{l} \alpha .
$$

Remark that for any formula $\alpha$, the sequent of the form $\mathrm{X}^{i} \alpha \Rightarrow \mathrm{X}^{i} \alpha$ is provable in $\mathrm{TN}[l]$. This can be shown by induction on $\alpha$. Thus, the sequents of the form $\mathrm{X}^{i} \alpha \Rightarrow \mathrm{X}^{i} \alpha$ can also be regarded as initial sequents.

Remark that $\mathrm{TN}[l]$ is just a logic parameterized by a fixed concrete positive integer $l$. Thus, before the detailed discussion, we have to fix $\mathrm{TN}[l]$ as a concrete logic such as TN[5]. Indeed, for example, $\mathrm{TN}[2]$ is different from $\mathrm{TN}[1]: p \wedge \mathrm{X} p \Rightarrow \mathrm{G} p$ is provable in $\mathrm{TN}[1]$, but it is not provable in $\mathrm{TN}[2]$. The unprovability of sequents is guaranteed by the cut-elimination theorem (Theorem 2.8).

Proposition 2.4. Let $m$ and $n$ be distinct fixed positive integers. The logics $\mathrm{TN}[m]$ and $\mathrm{TN}[n]$ are not theorem-equivalent.

Remark that the infinite-time version $\mathrm{TN}[\omega]$ of $\mathrm{TN}[l]$ is regarded as a non-commutative and intuitionistic version of Kawai's sequent calculus $\mathrm{LT}_{\omega}$ for (classical) linear-time temporal logic [12]. Since the treatment of the infinite rules $\left(\right.$ Gright $\left.^{\omega}\right)$ and $\left(\right.$ Fleft $\left.^{\omega}\right)$ is somewhat difficult, we do not know whether $\mathrm{TN}[\omega]$ is decidable or not. Such a problem is remained as an open question. In this paper, we do not discuss more about $\mathrm{TN}[\omega]$, since an aim of this paper is to obtain a decidable first-order logic.

In the following proposition, an expression $\alpha \Leftrightarrow \beta$ is used to denote an abbreviation of two sequents $\alpha \Rightarrow \beta$ and $\beta \Rightarrow \alpha$. 
Proposition 2.5. The following sequents are provable in $\mathrm{TN}[l]$ : for any formulas $\alpha, \beta$, any $i \in \omega$ and any positive integer $m$,

1. $X^{i} \sharp \Leftrightarrow \sharp$ where $\sharp \in\{\mathbf{1}, \top, \perp\}$,

2. $\mathrm{X}^{i}(\alpha \circ \beta) \Leftrightarrow \mathrm{X}^{i} \alpha \circ \mathrm{X}^{i} \beta$ where $\circ \in\{\rightarrow, \leftarrow, \wedge, \vee, *\}$,

3. $\mathrm{X}^{i} Q \alpha(x) \Leftrightarrow Q \mathrm{X}^{i} \alpha(x)$ where $Q \in\{\forall x, \exists x\}$,

4. $\mathrm{X}^{i} \sharp \alpha \Leftrightarrow \sharp \mathrm{X}^{i} \alpha$ where $\sharp \in\{\mathrm{G}, \mathrm{F}\}$,

5. $\mathrm{G} \alpha \Rightarrow \mathrm{F} \alpha$,

6. $\mathrm{G} \alpha \Rightarrow \mathrm{X} \alpha$,

7. $\mathrm{G} \alpha \Rightarrow \mathrm{XG} \alpha$,

8. $\mathrm{G} \alpha \Rightarrow \mathrm{GG} \alpha$,

9. $\mathrm{X}^{l+m} \alpha \Leftrightarrow \mathrm{X}^{l} \alpha$,

10. $\mathrm{G} \alpha \Leftrightarrow\left(\alpha \wedge \mathrm{X} \alpha \wedge \mathrm{X}^{2} \alpha \wedge \cdots \wedge \mathrm{X}^{l} \alpha\right)$,

11. $\mathrm{F} \alpha \Leftrightarrow\left(\alpha \vee \mathrm{X} \alpha \vee \mathrm{X}^{2} \alpha \vee \cdots \vee \mathrm{X}^{l} \alpha\right)$.

An expression like $\bigwedge\left\{\alpha_{i} \mid i \in \omega_{l}\right\}$ (or $\left.\bigvee\left\{\alpha_{i} \mid i \in \omega_{l}\right\}\right)$ where $\left\{\alpha_{i} \mid i \in \omega_{l}\right\}$ is a multiset means $\alpha_{0} \wedge \alpha_{1} \wedge \cdots \wedge \alpha_{l}$ (or $\alpha_{0} \vee \alpha_{1} \vee \cdots \vee \alpha_{l}$, respectively). For example, $\wedge\{\alpha, \alpha, \beta\}$ means $\alpha \wedge \alpha \wedge \beta$.

Definition 2.6. We fix a countable non-empty set $\Phi$ of atomic formulas, and define the sets $\Phi_{i}:=\left\{p_{i} \mid p \in \Phi\right\}(1 \leq i \in \omega)$ and $\Phi_{0}:=\Phi$ of atomic formulas. The language $\mathcal{L}_{\mathrm{TN}[l]}$ (or the set of formulas) of $\mathrm{TN}[l]$ is defined by using $\Phi, \mathbf{1}, \top, \perp, \rightarrow, \leftarrow, \wedge, \vee, *, \forall, \exists, \mathrm{X}, \mathrm{G}$ and $\mathrm{F}$. The language $\mathcal{L}_{\mathrm{FL}}$ of $\mathrm{FL}$ is defined by using $\bigcup_{i \in \omega} \Phi_{i}, \mathbf{1}, \top, \perp, \rightarrow, \leftarrow, \wedge, \vee, *, \forall$ and $\exists$.

A mapping $f$ from $\mathcal{L}_{\mathrm{TN}[l]}$ to $\mathcal{L}_{\mathrm{FL}}$ is defined by: for any $i \in \omega$ and any positive integer $m$,

1. $f\left(\mathrm{X}^{i} p\right):=p_{i} \in \Phi_{i}$ for any $p \in \Phi$ (especially, $\left.f(p):=p \in \Phi_{0}\right)$,

2. $f\left(\mathrm{X}^{i} \sharp\right):=\sharp$ where $\sharp \in\{\mathbf{1}, \top, \perp\}$,

3. $f\left(\mathrm{X}^{i}(\alpha \circ \beta)\right):=f\left(\mathrm{X}^{i} \alpha\right) \circ f\left(\mathrm{X}^{i} \beta\right)$ where $\circ \in\{\rightarrow, \leftarrow, \wedge, \vee, *\}$,

4. $f\left(\mathrm{X}^{i} Q \alpha(x)\right):=Q f\left(\mathrm{X}^{i} \alpha(x)\right)$ where $Q \in\{\forall x, \exists x\}$,

5. $f\left(\mathrm{X}^{l+m} \alpha\right):=f\left(\mathrm{X}^{l} \alpha\right)$,

6. $f\left(\mathrm{X}^{i} \mathrm{G} \alpha\right):=\bigwedge\left\{f\left(\mathrm{X}^{i+j} \alpha\right) \mid j \in \omega_{l}\right\}$,

7. $f\left(\mathrm{X}^{i} \mathrm{~F} \alpha\right):=\bigvee\left\{f\left(\mathrm{X}^{i+j} \alpha\right) \mid j \in \omega_{l}\right\}$. 
An expression $f(\Gamma)$ denotes the result of replacing every occurrence of a formula $\alpha$ in $\Gamma$ by an occurrence of $f(\alpha)$.

Strictly speaking, the embedding function $f$ is strongly dependent on the time bound $l$, i.e., $f$ should be denoted as $f_{l}$. Indeed, $f_{3}(\mathrm{G} p)$ and $f_{5}(\mathrm{G} p)$ are different. But, for the sake of brevity, a simple expression $f$ will be used in the following.

Theorem 2.7 (Embedding). Let $\Gamma$ be a sequence of formulas in $\mathcal{L}_{\mathrm{TN}[l]}, \gamma$ be a formula in $\mathcal{L}_{\mathrm{TN}[l]}$, and $f$ be the mapping defined in Definition 2.6. Then:

1. $\mathrm{TN}[l] \vdash \Gamma \Rightarrow \gamma$ iff FL $\vdash f(\Gamma) \Rightarrow f(\gamma)$.

2. $\mathrm{TN}[l]-$ (cut) $\vdash \Gamma \Rightarrow \gamma$ iff $\mathrm{FL}-($ cut $) \vdash f(\Gamma) \Rightarrow f(\gamma)$.

Proof. Since the case (2) can be obtained as a subproof of the case (1), we consider only (1).

" $(\Rightarrow)$ " By induction on a proof $P$ of $\Gamma \Rightarrow \gamma$ in TN $[l]$. We distinguish the cases according to the last inference of $P$, and show some cases.

Case $\left(\mathrm{X}^{i} p \Rightarrow \mathrm{X}^{i} p\right)$ : The last inference of $P$ is of the form: $\mathrm{X}^{i} p \Rightarrow \mathrm{X}^{i} p$. In this case, we obtain FL $\vdash f\left(\mathrm{X}^{i} p\right) \Rightarrow f\left(\mathrm{X}^{i} p\right)$, i.e., $\mathrm{FL} \vdash p_{i} \Rightarrow p_{i}\left(p_{i} \in \Phi_{i}\right)$.

Case $(\rightarrow$ left). The last inference of $P$ is of the form:

$$
\frac{\Gamma \Rightarrow \mathrm{X}^{i} \alpha \quad \Sigma, \mathrm{X}^{i} \beta, \Delta \Rightarrow \gamma}{\Sigma, \mathrm{X}^{i}(\alpha \rightarrow \beta), \Gamma, \Delta \Rightarrow \gamma}(\rightarrow \mathrm{left})
$$

By induction hypothesis, we have FL $\vdash f(\Gamma) \Rightarrow f\left(\mathrm{X}^{i} \alpha\right)$ and $\mathrm{FL} \vdash f(\Sigma)$, $f\left(\mathrm{X}^{i} \beta\right), f(\Delta) \Rightarrow f(\gamma)$. Then we obtain the required fact:

$$
\begin{array}{cc}
\vdots & \vdots \\
\frac{f(\Gamma) \Rightarrow f\left(\mathrm{X}^{i} \alpha\right)}{f(\Sigma), f\left(\mathrm{X}^{i} \alpha\right) \rightarrow f\left(\mathrm{X}^{i} \beta\right), f(\Gamma), f(\Delta) \Rightarrow f(\gamma)} & f\left(\mathrm{X}^{i} \beta\right), f(\Delta) \Rightarrow f(\gamma)
\end{array}
$$

where $f\left(\mathrm{X}^{i} \alpha\right) \rightarrow f\left(\mathrm{X}^{i} \beta\right)$ coincides with $f\left(\mathrm{X}^{i}(\alpha \rightarrow \beta)\right)$ by the definition of $f$.

Case (Xleft). The last inference of $P$ is of the form:

$$
\frac{\Gamma, \mathrm{X}^{l} \alpha, \Delta \Rightarrow \gamma}{\Gamma, \mathrm{X}^{l+m} \alpha, \Delta \Rightarrow \gamma} \text { (Xleft). }
$$

By induction hypothesis, we obtain the required fact: $\mathrm{FL} \vdash f(\Gamma), f\left(\mathrm{X}^{l} \alpha\right)$, $f(\Delta) \Rightarrow f(\gamma)$ where $f\left(\mathrm{X}^{l} \alpha\right)$ coincides with $f\left(\mathrm{X}^{l+m} \alpha\right)$ by the definition of $f$. 
Case (Gleft). The last inference of $P$ is of the form:

$$
\frac{\Gamma, \mathrm{X}^{i+k} \alpha, \Delta \Rightarrow \gamma}{\Gamma, \mathrm{X}^{i} \mathrm{G} \alpha, \Delta \Rightarrow \gamma}(\mathrm{Gleft})
$$

By induction hypothesis, we have FL $\vdash f(\Gamma), f\left(\mathrm{X}^{i+k} \alpha\right), f(\Delta) \Rightarrow f(\gamma)$, and hence obtain:

$$
\begin{gathered}
\vdots \\
f(\Gamma), f\left(\mathrm{X}^{i+k} \alpha\right), f(\Delta) \Rightarrow f(\gamma) \\
\vdots \\
\left(\wedge \operatorname{left} 1^{F L}\right) \text { and }\left(\wedge \operatorname{left} 2^{F L}\right) \\
f(\Gamma), \wedge\left\{f\left(\mathrm{X}^{i+j} \alpha\right) \mid j \in \omega_{l}\right\}, f(\Delta) \Rightarrow f(\gamma)
\end{gathered}
$$

where $\bigwedge\left\{f\left(\mathrm{X}^{i+j} \alpha\right) \mid j \in \omega_{l}\right\}$ coincides with $f\left(\mathrm{X}^{i} \mathrm{G} \alpha\right)$ by the definition of $f$, and $f\left(\mathrm{X}^{i+k} \alpha\right) \in\left\{f\left(\mathrm{X}^{i+j} \alpha\right) \mid j \in \omega_{l}\right\}$. Remark that the case $i>l$ is also included in this proof. In such a case, $f\left(\mathrm{X}^{i+k} \alpha\right)$ and $\bigwedge\left\{f\left(\mathrm{X}^{i+j} \alpha\right) \mid j \in \omega_{l}\right\}$ mean $f\left(\mathrm{X}^{l} \alpha\right)$ and $\overbrace{f\left(\mathrm{X}^{l} \alpha\right) \wedge f\left(\mathrm{X}^{l} \alpha\right) \wedge \cdots \wedge f\left(\mathrm{X}^{l} \alpha\right)}^{l}$, respectively.

Case (Gright). The last inference of $P$ is of the form:

$$
\frac{\left\{\Gamma \Rightarrow \mathrm{X}^{i+j} \alpha\right\}_{j \in \omega_{l}}}{\Gamma \Rightarrow \mathrm{X}^{i} \mathrm{G} \alpha} \text { (Gright). }
$$

By induction hypothesis, we have $\mathrm{FL} \vdash f(\Gamma) \Rightarrow f\left(\mathrm{X}^{i+j} \alpha\right)$ for all $j \in \omega_{l}$. Let $\Phi$ be the multiset $\left\{f\left(\mathrm{X}^{i+j} \alpha\right) \mid j \in \omega_{l}\right\}$. We obtain

$$
\begin{gathered}
\vdots \\
\left\{f(\Gamma) \Rightarrow f\left(\mathrm{X}^{i+j} \alpha\right)\right\} f\left(\mathrm{X}^{i+j} \alpha\right) \in \Phi \\
\vdots \\
f(\Gamma) \stackrel{\left(\operatorname{right}^{F L}\right)}{\Rightarrow} \wedge \Phi
\end{gathered}
$$

where $\bigwedge \Phi$ coincides with $f\left(\mathrm{X}^{i} \mathrm{G} \alpha\right)$ by the definition of $f$.

" $(\Leftarrow)$ " By induction on a proof $Q$ of $f(\Gamma) \Rightarrow f(\gamma)$ in FL. We distinguish the cases according to the last inference of $Q$, and show only the following case.

Case $\left(\wedge \operatorname{right}{ }^{F L}\right)$. The last inference of $Q$ is of the form:

$$
\frac{f(\Gamma) \Rightarrow f\left(\mathrm{X}^{i} \alpha\right) \quad f(\Gamma) \Rightarrow f\left(\mathrm{X}^{i} \beta\right)}{f(\Gamma) \Rightarrow f\left(\mathrm{X}^{i} \alpha\right) \wedge f\left(\mathrm{X}^{i} \beta\right)}\left(\operatorname{right}^{F L}\right)
$$


where $f\left(\mathrm{X}^{i}(\alpha \wedge \beta)\right)$ coincides with $f\left(\mathrm{X}^{i} \alpha\right) \wedge f\left(\mathrm{X}^{i} \beta\right)$ by the definition of $f$. By induction hypothesis, we have $\mathrm{TN}[l] \vdash \Gamma \Rightarrow \mathrm{X}^{i} \alpha$ and $\mathrm{TN}[l] \vdash \Gamma \Rightarrow \mathrm{X}^{i} \beta$. Then, we obtain the required fact:

$$
\frac{\Gamma \Rightarrow \mathrm{X}^{i} \alpha}{\Gamma \Rightarrow \mathrm{X}^{i} \beta} \quad(\wedge \text { right }) .
$$

Theorem 2.8 (Cut-elimination). The rule (cut) is admissible in cut-free $\mathrm{TN}[l]$.

Proof. Suppose TN $[l] \vdash \Gamma \Rightarrow \gamma$. Then, we have FL $\vdash f(\Gamma) \Rightarrow f(\gamma)$ by Theorem 2.7 (1), and hence FL - (cut) $\vdash f(\Gamma) \Rightarrow f(\gamma)$ by the well-known cut-elimination theorem for FL. By Theorem 2.7 (2), we obtain TN $[l]-$ (cut) $\vdash \Gamma \Rightarrow \gamma$.

It is known that some first-order logics without contraction rule are decidable. For example, it was shown by Komori [13] that some first-order intuitionistic substructural logics without the contraction rule, including FL, are decidable. For a review of the decision problems for substructural logics, see e.g., [10].

Theorem 2.9 (Decidability). TN $[l]$ is decidable.

Proof. By Theorem 2.7, the provability of TN $[l]$ can finitely be transformed into that of FL. Since FL is decidable, TN[l] is also decidable.

\section{Phase semantics}

The phase semantics for linear logics was originally introduced by Girard [5], and the phase semantics for intuitionistic non-commutative linear logics (including FL) was proposed by Abrusci [1]. A phase-semantic cut-elimination and completeness proof was proposed by Okada [16]. In this paper, we obtain a phase semantics for the propositional fragment of $\mathrm{TN}[l]$ based on an extension of Abrusci's non-commutative phase semantics, and prove the completeness theorem with respect to this semantics by using an extension of Okada's method.

Although the completeness theorem for the first-order predicate $\mathrm{TN}[l]$ can similarly be obtained based on an extension of Okada's method, such a theorem and its proof are omitted here, since the essential part of the 
semantics, i.e., the part of the temporal connectives, is completely included in the propositional part. In this section, we thus use the same name $\mathrm{TN}[l]$ for its propositional fragment.

The difference between the semantics for $\mathrm{TN}[l]$ and the semantics for FL is the definition of the valuations: Whereas the semantics for FL has a valuation $v$, the semantics for $\mathrm{TN}[l]$ has an infinite number of valuations $v^{i}$ $(i \in \omega)$, where $v^{0}$ is the same as $v$.

DeFinition 3.10. An intuitionistic phase space $^{1}$ is a structure $\langle\mathbf{M}$, cl $\rangle$ satisfying the following conditions:

1. $\mathbf{M}:=\langle M, \cdot, 1\rangle$ is a monoid with the identity 1 ,

2. $\mathrm{cl}$ is an operation on the powerset $P(M)$ of $M$ such that, for any $X, Y \in$ $P(M)$,

$\mathrm{C} 1: X \subseteq \operatorname{cl}(X)$,

$\mathrm{C} 2: \operatorname{clcl}(X) \subseteq \operatorname{cl}(X)$,

C3: $X \subseteq Y$ implies $\operatorname{cl}(X) \subseteq \operatorname{cl}(Y)$,

C4: $\operatorname{cl}(X) \circ \operatorname{cl}(Y) \subseteq \operatorname{cl}(X \circ Y)$

where the operation $\circ$ on $P(M)$ is defined as $X \circ Y:=\{x \cdot y \mid x \in X$ and $y \in Y\}$. The operation $c l$ is called here closure operation.

In order to obtain an interpretation of the logical constants and connectives, the corresponding constants and operations on $P(M)$ are defined below.

Definition 3.11. Constants and operations on $P(M)$ are defined as follows: for any $X, Y \in P(M)$,

1. $\mathbf{i}:=\operatorname{cl}\{1\}$,

2. $\dot{\top}:=M$,

3. $\dot{\perp}:=\operatorname{cl}(\emptyset)$,

4. $X \dot{\rightarrow}:=\{y \mid \forall x \in X(x \cdot y \in Y)\}$,

5. $X \leftarrow Y:=\{y \mid \forall x \in X(y \cdot x \in Y)\}$,

6. $X * Y:=\operatorname{cl}(X \circ Y)$,

\footnotetext{
${ }^{1}$ For the sake of brevity, the term "non-commutative" in the intuitionistic noncommutative phase space or structure is omitted in this paper.
} 
7. $X \dot{\wedge} Y:=X \cap Y$,

8. $X \dot{\vee} Y:=\operatorname{cl}(X \cup Y)$.

We define $D:=\{X \in P(M) \mid X=\operatorname{cl}(X)\}$. Then,

$$
\mathbf{D}:=\langle D, \dot{\rightarrow}, \dot{\leftarrow}, \dot{*}, \dot{\wedge}, \dot{\vee}, \dot{\mathbf{1}}, \dot{\top}, \dot{\perp}\rangle
$$

is called an intuitionistic phase structure.

Remark that the following facts hold: for any $X, X^{\prime}, Y, Y^{\prime}, Z \in P(M)$,

1. $X \subseteq Y \rightarrow Z$ iff $X \circ Y \subseteq Z$,

2. $X \subseteq Y \leftarrow Z$ iff $Y \circ X \subseteq Z$,

3. $X \subseteq X^{\prime}$ and $Y \subseteq Y^{\prime}$ imply $X \circ Y \subseteq X^{\prime} \circ Y^{\prime}, X^{\prime} \rightarrow Y \subseteq X \rightarrow Y^{\prime}$ and $X^{\prime} \leftarrow Y \subseteq X \leftarrow Y^{\prime}$.

Remark that $D$ is closed under the operations $\dot{\rightarrow}, \dot{\leftarrow}, \dot{*}, \dot{\wedge}, \dot{\vee}$ and $\bigcap$ (infinite meet), and that $\dot{\mathbf{i}}, \dot{\mathrm{T}}, \dot{\perp} \in D$.

DeFinition 3.12. Timed valuations $v^{i}$ for all $i \in \omega$ on an intuitionistic phase structure $\mathbf{D}:=\langle D, \dot{\rightarrow}, \dot{\leftarrow}, \dot{*}, \dot{\wedge}, \dot{V}, \dot{\mathbf{i}}, \dot{\top}, \dot{\perp}\rangle$ are mappings from the set of all propositional variables to $D$. Then, $v^{i}$ for all $i \in \omega$ are extended to mappings from the set $\Phi$ of all formulas to $D$ by: for any positive integer $m$,

1. $v^{i}(\mathbf{1}):=\mathbf{i}$,

2. $v^{i}(\top):=\dot{\top}$,

3. $v^{i}(\perp):=\dot{\perp}$,

4. $v^{i}(\alpha \rightarrow \beta):=v^{i}(\alpha) \dot{\rightarrow} v^{i}(\beta)$,

5. $v^{i}(\alpha \leftarrow \beta):=v^{i}(\alpha) \leftarrow v^{i}(\beta)$,

6. $v^{i}(\alpha * \beta):=v^{i}(\alpha) \dot{*} v^{i}(\beta)$,

7. $v^{i}(\alpha \wedge \beta):=v^{i}(\alpha) \dot{\wedge} v^{i}(\beta)$,

8. $v^{i}(\alpha \vee \beta):=v^{i}(\alpha) \dot{\vee} v^{i}(\beta)$,

9. $v^{i}(\mathrm{X} \alpha):=v^{i+1}(\alpha)$,

10. $v^{i}\left(\mathrm{X}^{l+m} \alpha\right):=v^{i}\left(\mathrm{X}^{l} \alpha\right)$,

11. $v^{i}(\mathrm{G} \alpha):=\bigcap_{j \in \omega_{l}} v^{i+j}(\alpha)$ if $i<l, v^{i}(\mathrm{G} \alpha):=v^{l}(\alpha)$ otherwise,

12. $v^{i}(\mathrm{~F} \alpha):=c l\left(\bigcup_{j \in \omega_{l}} v^{i+j}(\alpha)\right)$ if $i<l, v^{i}(\mathrm{~F} \alpha):=v^{l}(\alpha)$ otherwise. 
Remark that the following conditions hold: for any positive integer $m$,

1. $v^{l+m}(\alpha)=v^{0}\left(\mathrm{X}^{l+m} \alpha\right)=v^{0}\left(\mathrm{X}^{l} \alpha\right)=v^{l}(\alpha)$,

2. $v^{l+m}(\mathrm{G} \alpha)=v^{l}(\alpha)=v^{l+m}(\mathrm{~F} \alpha)$.

Definition 3.13. An intuitionistic timed phase model is a structure $\langle\mathbf{D}$, $\left.\left\{v^{i}\right\}_{i \in \omega}\right\rangle$ such that $\mathbf{D}$ is an intuitionistic phase structure, and $\left\{v^{i}\right\}_{i \in \omega}$ is a set of timed valuations. A formula $\alpha$ is true in an intuitionistic timed phase model $\left\langle\mathbf{D},\left\{v^{i}\right\}_{i \in \omega}\right\rangle$ if $\mathbf{i} \subseteq v^{0}(\alpha)$ (or equivalently $1 \in v^{0}(\alpha)$ ) holds, and valid in an intuitionistic phase structure $\mathbf{D}$ if it is true for any timed valuations $\left\{v^{i}\right\}_{i \in \omega}$ on the intuitionistic phase structure. A sequent $\alpha_{1}, \cdots, \alpha_{n} \Rightarrow \beta$ (or $\Rightarrow \beta$ ) is true in an intuitionistic timed phase model $\left\langle\mathbf{D},\left\{v^{i}\right\}_{i \in \omega}\right\rangle$ if the formula $\alpha_{1} * \cdots * \alpha_{n} \rightarrow \beta$ (or $\beta$ ) is true in it, and valid in an intuitionistic phase structure if so is $\alpha_{1} * \cdots * \alpha_{n} \rightarrow \beta$ (or $\beta$ ).

An expression $\Delta^{*}$ means $\delta_{1} * \cdots * \delta_{n}$ (or $\left.\emptyset\right)$ if $\Delta \equiv\left\langle\delta_{1}, \ldots, \delta_{n}\right\rangle(1 \leq n)$ (or $\Delta \equiv \emptyset$, respectively).

Theorem 3.14 (Soundness). If a sequent $S$ is provable in $\mathrm{TN}[l]$, then $S$ is valid for any intuitionistic phase structures.

Proof. By induction on a proof $P$ of $S$. We distinguish the cases according to the last inference of $P$. The cases for the logical inference rules (and initial sequents) excepting the cases for (Xleft), (Xright), (Gleft), (Gright), (Fleft) and (Fright) are similar to the cases in FL. The cases for (Xleft) and (Xright) are obvious by the definition of valuations. The cases for (Gright) and (Gleft), except the cases depending on the definition of valuations, are similar to the cases (Fleft) and (Fright), respectively. We thus show only the following cases.

Case (Gright): The last inference of $P$ is of the form:

$$
\frac{\left\{\Gamma \Rightarrow \mathrm{X}^{i+j} \alpha\right\}_{j \in \omega_{l}}}{\Gamma \Rightarrow \mathrm{X}^{i} \mathrm{G} \alpha} \text { (Gright). }
$$

Subcase $(i<l)$ : We show only the case for $\Gamma \neq \emptyset$. It is sufficient to show that if $\forall j \in \omega_{l}\left[1 \in v^{0}\left(\Gamma^{*} \rightarrow \mathrm{X}^{i+j} \alpha\right)\right]$ then $1 \in v^{0}\left(\Gamma^{*} \rightarrow \mathrm{X}^{i} \mathrm{G} \alpha\right)$. Suppose $\forall j \in$ $\omega_{l}\left[1 \in v^{0}\left(\Gamma^{*} \rightarrow \mathrm{X}^{i+j} \alpha\right)\right]$, i.e., $\forall x\left[x \in v^{0}\left(\Gamma^{*}\right)\right.$ implies $\left.\forall j \in \omega_{l}\left(x \in v^{0}\left(\mathrm{X}^{i+j} \alpha\right)\right)\right]$ where $x \in v^{0}\left(\mathrm{X}^{i+j} \alpha\right)=v^{i+j}(\alpha)$. We show $1 \in v^{0}\left(\Gamma^{*} \rightarrow \mathrm{X}^{i} \mathrm{G} \alpha\right)$, i.e., $\forall x[x \in$ $v^{0}\left(\Gamma^{*}\right)$ implies $\left.x \in v^{0}\left(\mathrm{X}^{i} \mathrm{G} \alpha\right)\right]$ where $x \in v^{0}\left(\mathrm{X}^{i} \mathrm{G} \alpha\right)=\bigcap_{j \in \omega_{l}} v^{i+j}(\alpha)$ iff $\forall j \in$ $\omega_{l}\left[x \in v^{i+j}(\alpha)\right]$. Suppose $x \in v^{0}\left(\Gamma^{*}\right)$. Then we have $\forall j \in \omega_{l}\left[x \in v^{i+j}(\alpha)\right]$ by the induction hypothesis. 
Subcase $(i \geq l)$ : We show only the case for $\Gamma \neq \emptyset$. Let $i=l+m$ where $m \geq 0$. In this case, we can proceed the proof by the same manner as in the case $i<l$. Thus, it is sufficient to show the fact that $\forall j \in \omega_{l}$ $\left[x \in v^{0}\left(\mathrm{X}^{l+m+j} \alpha\right)\right]$ implies $x \in v^{0}\left(\mathrm{X}^{l+m} \mathrm{G} \alpha\right)$. This fact can be shown by the fact $v^{0}\left(\mathrm{X}^{l+m+j} \alpha\right)=v^{l}(\alpha)=v^{0}\left(\mathrm{X}^{l+m} \mathrm{G} \alpha\right)$.

Case (Gleft): The last inference of $P$ is of the form: for any $k \in \omega_{l}$,

$$
\frac{\Delta, \mathrm{X}^{i+k} \alpha, \Gamma \Rightarrow \gamma}{\Delta, \mathrm{X}^{i} \mathrm{G} \alpha, \Gamma \Rightarrow \gamma}(\text { Gleft })
$$

Subcase $(i<l)$ : We only show the case for $\Gamma \neq \emptyset$. It is sufficient to show that if $1 \in v^{0}\left(\Delta^{*} * \mathrm{X}^{i+k} \alpha \rightarrow\left(\Gamma^{*} \rightarrow \gamma\right)\right)$, then $1 \in v^{0}\left(\Delta^{*} * \mathrm{X}^{i} \mathrm{G} \alpha \rightarrow\left(\Gamma^{*} \rightarrow \gamma\right)\right)$, since $\Delta^{*} * \mathrm{X}^{i+k} \alpha \rightarrow\left(\Gamma^{*} \rightarrow \gamma\right)$ and $\Delta^{*} * \mathrm{X}^{i} \mathrm{G} \alpha \rightarrow\left(\Gamma^{*} \rightarrow \gamma\right)$ are logically equivalent to $\Delta^{*} * \mathrm{X}^{i+k} \alpha * \Gamma^{*} \rightarrow \gamma$ and $\Delta^{*} * \mathrm{X}^{i} \mathrm{G} \alpha * \Gamma^{*} \rightarrow \gamma$, respectively. Suppose $1 \in v^{0}\left(\Delta^{*} *\right.$ $\left.\mathrm{X}^{i+k} \alpha \rightarrow\left(\Gamma^{*} \rightarrow \gamma\right)\right)$, i.e., $1 \in v^{0}\left(\Delta^{*} * \mathrm{X}^{i+k}\right) \dot{\rightarrow} v^{0}\left(\Gamma^{*} \rightarrow \gamma\right)$ iff $1 \in\left\{y \mid \forall x \in v^{0}\left(\Delta^{*} *\right.\right.$ $\left.\mathrm{X}^{i+k} \alpha\right)\left(x \cdot y \in v^{0}\left(\Gamma^{*} \rightarrow \gamma\right)\right\}$ iff $\forall x\left[x \in v^{0}\left(\Delta^{*} * \mathrm{X}^{i+k} \alpha\right)\right.$ implies $\left.x \in v^{0}\left(\Gamma^{*} \rightarrow \gamma\right)\right]$. We show $1 \in v^{0}\left(\Delta^{*} * \mathrm{X}^{i} \mathrm{G} \alpha \rightarrow\left(\Gamma^{*} \rightarrow \gamma\right)\right)$, i.e., $\forall x\left[x \in v^{0}\left(\Delta^{*} * \mathrm{X}^{i} \mathrm{G} \alpha\right)\right.$ implies $\left.x \in v^{0}\left(\Gamma^{*} \rightarrow \gamma\right)\right]$. To show this, it is sufficient to show $v^{0}\left(\Delta^{*} * \mathrm{X}^{i} \mathrm{G} \alpha\right) \subseteq$ $v^{0}\left(\Delta^{*} * \mathrm{X}^{i+k} \alpha\right)$ by the hypothesis. Also, to show $v^{0}\left(\Delta^{*} * \mathrm{X}^{i} \mathrm{G} \alpha\right) \subseteq v^{0}\left(\Delta^{*} *\right.$ $\left.\mathrm{X}^{i+k} \alpha\right)$, it is sufficient to show $v^{0}\left(\mathrm{X}^{i} \mathrm{G} \alpha\right) \subseteq v^{0}\left(\mathrm{X}^{i+k} \alpha\right)$. This can be shown as $v^{0}\left(\mathrm{X}^{i} \mathrm{G} \alpha\right)=v^{i}(\mathrm{G} \alpha)=\bigcap_{j \in \omega_{l}} v^{i+j}(\alpha) \subseteq v^{i+k}(\alpha)=v^{0}\left(\mathrm{X}^{i+k} \alpha\right)$.

Subcase $(i \geq l)$ : We show only the case for $\Gamma \neq \emptyset$. Let $i=l+m$ where $m \geq 0$. In this case, we can proceed the proof by the same manner as in the case $i<l$. Thus, it is sufficient to show the fact $v^{0}\left(\mathrm{X}^{l+m} \mathrm{G} \alpha\right) \subseteq v^{0}\left(\mathrm{X}^{l+m+k} \alpha\right)$. This fact is obvious since $v^{0}\left(\mathrm{X}^{l+m} \mathrm{G} \alpha\right)=v^{l}(\alpha)=v^{0}\left(\mathrm{X}^{l+m+k} \alpha\right)$.

In order to prove the strong completeness theorem, we have to construct a canonical model.

For the sake of clearlity for the completeness proof, an expression $[\Gamma]$ is used to explicitly represent a sequence of formulas, i.e., $[\Gamma]$ and $\Gamma$ are identical, but only the expressions are different.

Definition 3.15. We define a monoid $\langle M, \cdot, 1\rangle$ as follows:

1. $M:=\{[\Gamma] \mid[\Gamma]$ is a finite sequence of formulas $\}$,

2. $[\Gamma] \cdot[\Delta]:=[\Gamma, \Delta]$ (the concatenation),

$3.1:=[]$ (the empty sequence).

We define the following: for any $i \in \omega$ and any formula $\alpha$,

$$
\|\alpha\|^{i}:=\left\{[\Gamma] \mid \vdash_{\mathrm{cf}} \Gamma \Rightarrow \mathrm{X}^{i} \alpha\right\}
$$

where $\vdash_{\text {cf }}$ means "provable in cut-free $\mathrm{TN}[l]$ ". 
Definition 3.16. We define

$$
D:=\left\{X \mid X=\bigcap_{i \in I}\left\|\alpha_{i}\right\|^{0}\right\}
$$

for an arbitrary (non-empty) indexing set $I$ and an arbitrary formula $\alpha_{i}$. We then define

$$
\operatorname{cl}(X):=\bigcap\{Y \in D \mid X \subseteq Y\} .
$$

We define the following constants and operations on $P(M)$ : for any $X, Y \in$ $P(M)$

1. $\mathrm{i}:=\operatorname{cl}\{1\}$,

2. $\dot{\top}:=M$,

3. $\dot{\perp}:=\operatorname{cl}(\emptyset)$,

4. $X \dot{\rightarrow}:=\{[\Delta] \mid \forall[\Gamma] \in X([\Delta, \Gamma] \in Y)\}$,

5. $X \leftarrow Y:=\{[\Delta] \mid \forall[\Gamma] \in X([\Gamma, \Delta] \in Y)\}$,

6. $X * Y:=\operatorname{cl}(X \circ Y)$ where $X \circ Y:=\{[\Gamma, \Delta] \mid[\Gamma] \in X$ and $[\Delta] \in Y\}$,

7. $X \dot{\wedge} Y:=X \cap Y$,

8. $X \dot{\vee} Y:=\operatorname{cl}(X \cup Y)$.

Timed valuations $v^{i}$ for all $i \in \omega$ are mappings from the set of all propositional variables to $D$ such that $v^{i}(p):=\|p\|^{i}$.

We have the following facts: for any $X, Y, Z \in P(M)$,

1. $X \subseteq Y \rightarrow Z$ iff $X \circ Y \subseteq Z$,

2. $X \subseteq Y \leftarrow Z$ iff $Y \circ X \subseteq Z$.

Remark that $D$ is closed under arbitrary $\bigcap$.

Lemma 3.17. Let $D$ be $\left\{X \mid X=\bigcap_{i \in I}\left\|\alpha_{i}\right\|^{0}\right\}$, and $D_{c}$ be $\{X \in P(M) \mid X=$ $\operatorname{cl}(X)\}$. Then, $D=D_{c}$.

Proof. First, we show $D_{c} \subseteq D$. Suppose $X \in D_{c}$. Then $X=\operatorname{cl}(X)=$ $\bigcap\{Y \in D \mid X \subseteq Y\} \in D$. Next, we show $D \subseteq D_{c}$. Suppose $X \in D$. We show $X \in D_{c}$, i.e. $X=\bigcap\{Y \in D \mid X \subseteq Y\}$. To show this, it is sufficient to prove that

(1) $X \subseteq\{[\Gamma] \mid \forall W[W \in D$ and $X \subseteq W$ imply $[\Gamma] \in W]\}$,

(2) $\{[\Gamma] \mid \forall W[W \in D$ and $X \subseteq W$ imply $[\Gamma] \in W]\} \subseteq X$. 
First, we show (1). Suppose $[\Delta] \in X$ and asuume $W \in D$ and $X \subseteq W$ for any $W$. Then we have $[\Delta] \in X \subseteq W$. Next we show (2). Suppose $[\Delta] \in\{[\Gamma] \mid \forall W[W \in D$ and $X \subseteq W$ imply $[\Gamma] \in W]\}$. By the assumption $X \in D$ and the fact that $X \subseteq X$, we have $[\Delta] \in X$.

Lemma 3.18. For any $X, Y \subseteq M$, if $Y \in D$, then $X \dot{\rightarrow} Y, X \leftarrow Y \in D$.

Proof. We show only $X \dot{\rightarrow} Y \in D$. The case $X \dot{\leftarrow} Y \in D$ can similarly be shown. Before the proof, it is remarked that the following rules

$$
\frac{\Gamma \Rightarrow \mathrm{X}^{i}(\alpha \rightarrow \beta)}{\Gamma, \mathrm{X}^{i} \alpha \Rightarrow \mathrm{X}^{i} \beta}\left(\rightarrow \text { right }^{-1}\right) \quad \frac{\Gamma, \mathrm{X}^{i}(\alpha * \beta), \Delta \Rightarrow \gamma}{\Gamma, \mathrm{X}^{i} \alpha, \mathrm{X}^{i} \beta, \Delta \Rightarrow \gamma}\left(* \mathrm{eft}^{-1}\right)
$$

are admissible in cut-free $\mathrm{TN}[l]$.

Suppose $X \subseteq M$ and $Y \in D$. We have:

$$
\begin{aligned}
X \dot{\rightarrow} Y= & X \dot{\rightarrow} \bigcap_{i \in I}\left\|\alpha_{i}\right\|^{0} \\
= & \left\{[\Delta] \mid \forall[\Gamma] \in X\left([\Delta, \Gamma] \in\left\{[\Pi] \mid \forall i \in I\left([\Pi] \in\left\|\alpha_{i}\right\|^{0}\right)\right\}\right)\right\} \\
= & \left\{[\Delta] \mid \forall[\Gamma] \in X\left(\forall i \in I\left(\vdash_{\mathrm{cf}} \Delta, \Gamma \Rightarrow \alpha_{i}\right)\right)\right\} \\
= & \left\{[\Delta] \mid \forall[\Gamma] \in X\left(\forall i \in I\left(\vdash_{\mathrm{cf}} \Delta \Rightarrow \Gamma^{*} \rightarrow \alpha_{i}\right)\right)\right\} \\
& \left.\quad \quad \text { by using }(* \text { left }),\left(* \text { left }^{-1}\right),(\rightarrow \text { right }) \text { and }\left(\rightarrow \text { right }^{-1}\right)\right) \\
= & \left\{[\Delta] \mid \forall[\Gamma] \in X\left(\forall i \in I\left([\Delta] \in\left\|\Gamma^{*} \rightarrow \alpha_{i}\right\|^{0}\right)\right)\right\} \\
= & \bigcap\left\{\left\|\Gamma^{*} \rightarrow \alpha_{i}\right\|^{0} \mid i \in I \text { and }[\Gamma] \in X\right\} \in D .
\end{aligned}
$$

Then, we can show the following.

Proposition 3.19. The structure $\mathbf{D}:=\langle D, \dot{\rightarrow}, \dot{\leftarrow}, \dot{*}, \dot{\wedge}, \dot{\vee}, \dot{\mathbf{1}}, \dot{\top}, \dot{\perp}\rangle$ defined in Definition 3.16 forms an intuitionistic phase structure.

Proof. We can verify that $D$ is closed under $\dot{\rightarrow}, \dot{\leftarrow}, \dot{*}, \dot{\wedge}$ and $\dot{V}$. In particular, for $\dot{\rightarrow}$ and $\dot{\leftarrow}$, we use Lemma 3.18. The fact $\dot{\mathbf{1}}, \dot{\top}, \dot{\perp} \in D$ is obvious. We can verify that the conditions $\mathrm{C} 1-\mathrm{C} 4$ for the closure operation hold for this structure. The conditions $\mathrm{C} 1-\mathrm{C} 3$ are obvious. We only show $\mathrm{C} 4$ : $\operatorname{cl}(X) \circ \operatorname{cl}(Y) \subseteq \operatorname{cl}(X \circ Y)$ for any $X, Y \in P(M)$. We assume the following facts, which will be proved later: for any $X, Y \in P(M)$,

(*) $\operatorname{cl}(X) \cdot Y \subseteq \operatorname{cl}(X \circ Y)$,

$(* *) X \cdot \operatorname{cl}(Y) \subseteq \operatorname{cl}(X \circ Y)$. 
By using the facts $(*)$ and $(* *)$ and Lemma 3.17, we have:

$$
\operatorname{cl}(X) \circ \operatorname{cl}(Y) \subseteq \operatorname{cl}(\operatorname{cl}(X) \circ Y) \subseteq \operatorname{cl}(\operatorname{cl}(X \circ Y))=\operatorname{cl}(X \circ Y) .
$$

We show the remained facts $(*)$ and $(* *)$. We have $X \circ Y \subseteq \operatorname{cl}(X \circ Y)$ by the condition $\mathrm{C} 1$, and hence $X \subseteq Y \rightarrow \operatorname{cl}(X \circ Y)$ and $Y \subseteq X \leftarrow \operatorname{cl}(X \circ Y)$ hold. Moreover, by the condition $\mathrm{C} 3$, we have $\operatorname{cl}(X) \subseteq \operatorname{cl}(Y \rightarrow \operatorname{cl}(X \circ Y))$ and $\operatorname{cl}(Y) \subseteq \operatorname{cl}(X \leftarrow \operatorname{cl}(X \circ Y))$. Here, by $\operatorname{cl}(X \circ Y) \in D$ and Lemma 3.18, we have $Y \rightarrow \operatorname{cl}(X \circ Y) \in D$ and $X \leftarrow \operatorname{cl}(X \circ Y) \in D$. Thus, we obtain

$$
\begin{aligned}
& c l(X) \subseteq \operatorname{cl}(Y \dot{\rightarrow} c l(X \circ Y))=Y \dot{\rightarrow} c l(X \circ Y), \\
& c l(Y) \subseteq \operatorname{cl}(X \leftarrow \operatorname{cl}(X \circ Y))=X \dot{\leftarrow} \operatorname{cl}(X \circ Y)
\end{aligned}
$$

by Lemma 3.17. Therefore we obtain the required facts.

We then have a modified version of the key lemma by Okada [16].

Lemma 3.20. For any $i \in \omega$ and any formula $\alpha,\left[\mathrm{X}^{i} \alpha\right] \in v^{i}(\alpha) \subseteq\|\alpha\|^{i}$.

Proof. By induction on $\alpha$. The cases for $\alpha \equiv \mathbf{1}, \top, \perp, \beta \rightarrow \gamma, \beta \leftarrow \gamma, \beta * \gamma$, $\beta \wedge \gamma$ and $\beta \vee \gamma$ are similar to the cases in FL. We thus show only the following cases.

Case $(\alpha \equiv \mathrm{X} \beta)$ : By the hypothesis of induction, we have $\left[\mathrm{X}^{i+1} \beta\right] \in$ $v^{i+1}(\beta) \subseteq\|\beta\|^{i+1}$. We also have $\|\beta\|^{i+1}=\|\mathrm{X} \beta\|^{i}$ by the definition of $\|\cdot\|^{i}$. Thus, we obtain $\left[\mathrm{X}^{i}(\mathrm{X} \beta)\right] \in v^{i}(\mathrm{X} \beta) \subseteq\|\mathrm{X} \beta\|^{i}$.

Case $(\alpha \equiv \mathrm{G} \beta)$ :

Subcase $(i<l)$ : We show $\left[\mathrm{X}^{i} \mathrm{G} \beta\right] \in v^{i}(\mathrm{G} \beta) \subseteq\|\mathrm{G} \beta\|^{i}$ for any $i<l$. First, we show $\left[\mathrm{X}^{i} \mathrm{G} \beta\right] \in v^{i}(\mathrm{G} \beta)$, i.e., $\left[\mathrm{X}^{i} \mathrm{G} \beta\right] \in \bigcap_{j \in \omega_{l}} v^{i+j}(\beta)$, i.e., $\forall j \in \omega_{l}\left(\left[\mathrm{X}^{i} \mathrm{G} \beta\right] \in\right.$ $\left.v^{i+j}(\beta)\right)$. Since $v^{i+j}(\beta) \in D$, we have $v^{i+j}(\beta)=\bigcap_{k \in I}\left\|\delta_{k}\right\|^{0}=\{[\Gamma] \mid \forall k \in$ $\left.I\left(\vdash_{\mathrm{cf}} \Gamma \Rightarrow \delta_{k}\right)\right\}$. Thus, $\forall j \in \omega_{l}\left(\left[\mathrm{X}^{i} \mathrm{G} \beta\right] \in v^{i+j}(\beta)\right)$ means $(*): \forall k \in I\left(\vdash_{\mathrm{cf}}\right.$ $\left.\mathrm{X}^{i} \mathrm{G} \beta \Rightarrow \delta_{k}\right)$. On the other hand, by the hypothesis of induction, we have $\forall j \in \omega_{l}\left(\left[\mathrm{X}^{i+j} \beta\right] \in v^{i+j}(\beta)\right)$, i.e., $(* *): \forall k \in I \forall j \in \omega_{l}\left(\vdash_{\mathrm{cf}} \mathrm{X}^{i+j} \beta \Rightarrow \delta_{k}\right)$. By taking a positive integer $m$ for $j \in \omega_{l}$ in (**) and by applying (Gleft) to $(* *)$, we obtain $(*)$.

Next we show $v^{i}(\mathrm{G} \beta) \subseteq\|\mathrm{G} \beta\|^{i}$. Suppose $[\Gamma] \in v^{i}(\mathrm{G} \beta)$, i.e., $[\Gamma] \in$ $\bigcap_{j \in \omega_{l}} v^{i+j}(\beta)$. We show $[\Gamma] \in\|\mathrm{G} \beta\|^{i}$, i.e., $\vdash_{\mathrm{cf}} \Gamma \Rightarrow \mathrm{X}^{i} \mathrm{G} \beta$. By the hypothesis of induction, we have $v^{i+j}(\beta) \subseteq\|\beta\|^{i+j}$. Thus, we obtain $[\Gamma] \in \bigcap_{j \in \omega_{l}} v^{i+j}(\beta) \subseteq$ $\bigcap_{j \in \omega_{l}}\|\beta\|^{i+j}$, and hence $[\Gamma] \in \bigcap_{j \in \omega_{l}}\|\beta\|^{i+j}$, i.e., $\forall j \in \omega_{l}\left([\Gamma] \in\|\beta\|^{i+j}\right)$, i.e., $\forall j \in$ $\omega_{l}\left(\vdash_{\text {cf }} \Gamma \Rightarrow \mathrm{X}^{i+j} \beta\right)$. By applying (Gright) to this, we obtain $\vdash_{\mathrm{cf}} \Gamma \Rightarrow \mathrm{X}^{i} \mathrm{G} \beta$. 
Subcase $(i \geq l)$ : Let $i=l+m$ where $m \geq 0$. We show $\left[\mathrm{X}^{l+m} \mathrm{G} \beta\right] \in$ $v^{l+m}(\mathrm{G} \beta) \subseteq\|\mathrm{G} \beta\|^{l+m}$. First, we show that $\left[\mathrm{X}^{l+m} \mathrm{G} \beta\right] \in v^{l+m}(\mathrm{G} \beta)$, i.e., $\left[\mathrm{X}^{l+m} \mathrm{G} \beta\right] \in v^{l+m}(\mathrm{G} \beta)=v^{l}(\beta)$. Since $v^{l}(\beta) \in D$, we have $v^{l}(\beta)=\bigcap_{k \in I}\left\|\delta_{k}\right\|^{0}$ $=\left\{[\Gamma] \mid \forall k \in I\left(\vdash_{\mathrm{cf}} \Gamma \Rightarrow \delta_{k}\right)\right\}$. Thus, $\left[\mathrm{X}^{l+m} \mathrm{G} \beta\right] \in v^{l+m}(\mathrm{G} \beta)$ means $(*): \forall k \in$ $I\left(\vdash_{\mathrm{cf}} \mathrm{X}^{l+m} \mathrm{G} \beta \Rightarrow \delta_{k}\right)$. On the other hand, by the hypothesis of induction, we have $\left[\mathrm{X}^{l} \beta\right] \in v^{l}(\beta)$, i.e., $(* *): \forall k \in I\left(\vdash_{\mathrm{cf}} \mathrm{X}^{l} \beta \Rightarrow \delta_{k}\right)$. We thus obtain $(*)$ from $(* *)$ by using (Xleft) and (Gleft): for any $k \in I$,

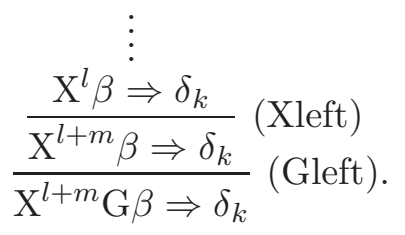

Next, we show $v^{l+m}(\mathrm{G} \beta) \subseteq\|\mathrm{G} \beta\|^{l+m}$. We have $v^{l+m}(\mathrm{G} \beta)=v^{l}(\beta) \subseteq\|\beta\|^{l}$ by the hypothesis of induction. Thus, it is sufficient to show $\|\beta\|^{l} \subseteq\|\mathrm{G} \beta\|^{l+m}$. We show this. Suppose $[\Gamma] \in\|\beta\|^{l}$, i.e., $\vdash_{\mathrm{cf}} \Gamma \Rightarrow \mathrm{X}^{l} \beta$. Then, we obtain $\vdash_{\text {cf }} \Gamma \Rightarrow \mathrm{X}^{l+m} \mathrm{G} \beta$ (i.e., $[\Gamma] \in\|\mathrm{G} \beta\|^{l+m}$ ) by using (Gright) and (Xright):

$$
\begin{array}{ccc}
\vdots & \vdots & \vdots \\
\frac{\Gamma \Rightarrow \mathrm{X}^{l} \beta}{\Gamma \Rightarrow \mathrm{X}^{l+m} \beta}(\mathrm{Xright}) & \frac{\Gamma \Rightarrow \mathrm{X}^{l} \beta}{\Gamma \Rightarrow \mathrm{X}^{l+m+1} \beta} \text { (Xright) } & \frac{\Gamma \Rightarrow \mathrm{X}^{l} \beta}{\Gamma \Rightarrow \mathrm{X}^{l+m+l} \beta} \\
\text { (Xright) } & \text { (Gright). }
\end{array}
$$

Case $(\alpha \equiv \mathrm{F} \beta)$ :

Subcase $(i<l)$ : We show $\left[\mathrm{X}^{i} \mathrm{~F} \beta\right] \in v^{i}(\mathrm{~F} \beta) \subseteq\|\mathrm{F} \beta\|^{i}$ for any $i<l$. First, we show $\left[\mathrm{X}^{i} \mathrm{~F} \beta\right] \in v^{i}(\mathrm{~F} \beta)$, i.e., $\left[\mathrm{X}^{i} \mathrm{~F} \beta\right] \in v^{i}(\mathrm{~F} \beta)=c l\left(\bigcup_{j \in \omega_{l}} v^{i+j}(\beta)\right)=\bigcap\{Y \in$ $\left.D \mid \bigcup_{j \in \omega_{l}} v^{i+j}(\beta) \subseteq Y\right\}$. Thus, we show

$$
\forall W\left[W \in D \text { and } \bigcup_{j \in \omega_{l}} v^{i+j}(\beta) \subseteq W \text { imply }\left[\mathrm{X}^{i} \mathrm{~F} \beta\right] \in W\right] .
$$

Suppose $W \in D$ and $\bigcup_{j \in \omega_{l}} v^{i+j}(\beta) \subseteq W$, and the induction hypotheses $\forall j \in$ $\omega_{l}\left(\left[\mathrm{X}^{i+j} \beta\right] \in v^{i+j}(\beta)\right)$. Then, we have: for any $j \in \omega_{l}$,

$$
\left[\mathrm{X}^{i+j} \beta\right] \in \bigcup_{j \in \omega_{l}} v^{i+j}(\beta) \subseteq W=\bigcap_{k \in I}\left\|\delta_{k}\right\|^{0}=\left\{[\Delta] \mid \forall k \in I\left([\Delta] \in\left\|\delta_{k}\right\|^{0}\right)\right\},
$$

and hence $\forall k \in I \forall j \in \omega_{l}\left(\vdash_{\mathrm{cf}} \mathrm{X}^{i+j} \beta \Rightarrow \delta_{k}\right)$. This implies $\forall k \in I\left(\vdash_{\mathrm{cf}}\right.$ $\mathrm{X}^{i} \mathrm{~F} \beta \Rightarrow \delta_{k}$ ) by (Fleft), and this means $\left[\mathrm{X}^{i} \mathrm{~F} \beta\right] \in \bigcap_{k \in I}\left\|\delta_{k}\right\|^{0}=W$. 
Second, we show $v^{i}(\mathrm{~F} \beta) \subseteq\|\mathrm{F} \beta\|^{i}$. Suppose $[\Gamma] \in v^{i}(\mathrm{~F} \beta)$. Then, we have $[\Gamma] \in \operatorname{cl}\left(\bigcup_{j \in \omega_{l}} v^{i+j}(\beta)\right)$, i.e.,

$$
\forall W\left[W \in D \text { and } \bigcup_{j \in \omega_{l}} v^{i+j}(\beta) \subseteq W \text { imply }[\Gamma] \in W\right] .
$$

We take $\|\mathrm{F} \beta\|^{i}$ for $W$. If we can show $\bigcup_{j \in \omega_{l}} v^{i+j}(\beta) \subseteq\|\mathrm{F} \beta\|^{i}$, then $[\Gamma] \in\|\mathrm{F} \beta\|^{i}$. Thus, we prove this. Suppose $[\Delta] \in \bigcup_{j \in \omega_{l}} v^{i+j}(\beta)$. Then, $[\Delta] \in \bigcup_{j \in \omega_{l}} v^{i+j}(\beta) \subseteq$ $\bigcup_{j \in \omega_{l}}\|\beta\|^{i+j}$ by the induction hypotheses, and hence we obtain $[\Delta] \in\|\beta\|^{i+k}$ for some $k \in \omega_{l}$, i.e., $\vdash_{\text {cf }} \Delta \Rightarrow \mathrm{X}^{i+k} \beta$. Thus, we obtain $\vdash_{\mathrm{cf}} \Delta \Rightarrow \mathrm{X}^{i} \mathrm{~F} \beta$ by (Fright). This means $[\Delta] \in\|\mathrm{F} \beta\|^{i}$.

Subcase $(i \geq l)$ : Let $i=l+m$ where $m \geq 0$. We show $\left[\mathrm{X}^{l+m} \mathrm{~F} \beta\right] \in$ $v^{l+m}(\mathrm{~F} \beta) \subseteq\|\mathrm{F} \beta\|^{l+m}$. First, we show that $\left[\mathrm{X}^{l+m} \mathrm{~F} \beta\right] \in v^{l+m}(\mathrm{~F} \beta)$, i.e., $\left[\mathrm{X}^{l+m} \mathrm{~F} \beta\right] \in v^{l+m}(\mathrm{~F} \beta)=v^{l}(\beta)$. Since $v^{l}(\beta) \in D$, we have $v^{l}(\beta)=\bigcap_{k \in I}\left\|\delta_{k}\right\|^{0}$ $=\left\{[\Gamma] \mid \forall k \in I\left(\vdash_{\mathrm{cf}} \Gamma \Rightarrow \delta_{k}\right)\right\}$. Thus, $\left[\mathrm{X}^{l+m} \mathrm{~F} \beta\right] \in v^{l+m}(\mathrm{~F} \beta)$ means $(*): \forall k \in$ $I\left(\vdash_{\mathrm{cf}} \mathrm{X}^{l+m} \mathrm{~F} \beta \Rightarrow \delta_{k}\right)$. On the other hand, by the hypothesis of induction, we have $\left[\mathrm{X}^{l} \beta\right] \in v^{l}(\beta)$, i.e., $(* *): \forall k \in I\left(\vdash_{\mathrm{cf}} \mathrm{X}^{l} \beta \Rightarrow \delta_{k}\right)$. We thus obtain $(*)$ from $(* *)$ by using (Xleft) and (Fleft): for any $k \in I$,

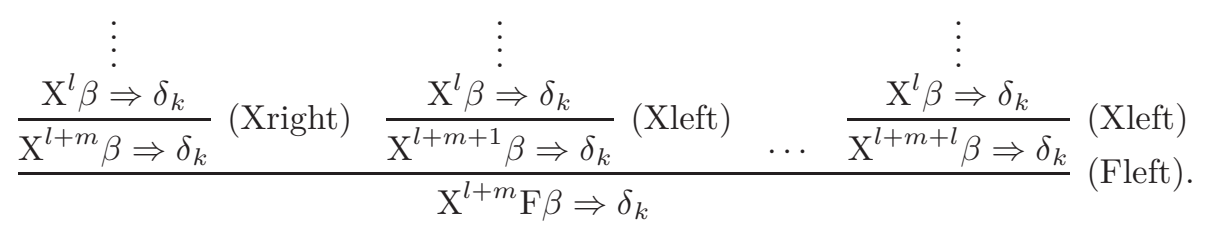

Next, we show $v^{l+m}(\mathrm{~F} \beta) \subseteq\|\mathrm{F} \beta\|^{l+m}$. We have $v^{l+m}(\mathrm{~F} \beta)=v^{l}(\beta) \subseteq\|\beta\|^{l}$ by the hypothesis of induction. Thus, it is sufficient to show $\|\beta\|^{l} \subseteq\|\mathrm{F} \beta\|^{l+m}$. We show this. Suppose $[\Gamma] \in\|\beta\|^{l}$, i.e., $\vdash_{\mathrm{cf}} \Gamma \Rightarrow \mathrm{X}^{l} \beta$. Then, we obtain $\vdash_{\mathrm{cf}} \Gamma \Rightarrow \mathrm{X}^{l+m} \mathrm{~F} \beta$ (i.e., $[\Gamma] \in\|\mathrm{F} \beta\|^{l+m}$ ) by using (Fright) and (Xright):

$$
\frac{\Gamma \Rightarrow \mathrm{X}^{l} \beta}{\Gamma \Rightarrow \mathrm{X}^{l+m} \beta} \text { (Xright) }
$$

Theorem 3.21 (Strong completeness). If a sequent $S$ is valid for any intuitionistic phase structures, then $S$ is provable in cut-free $\mathrm{TN}[l]$. 
Proof. Using Lemma 3.20, we can obtain this theorem as follows. If a formula $\alpha$, which corresponds to $S$, is true, then [ ] $\in v^{0}(\alpha)$. On the other hand $v^{i}(\alpha) \subseteq\|\alpha\|^{i}$ for any $i \in \omega$, and hence [ ] $\in\|\alpha\|^{0}$, which means " $\Rightarrow \alpha$ is provable in cut-free $\mathrm{TN}[l]$ ".

An alternative proof of the cut-elimination theorem for $\mathrm{TN}[l]$ is also obtained as follows. If a sequent $S$ is provable in $\operatorname{TN}[l]$, then $S$ is valid by Theorem 3.14. By Theorem 3.21, $S$ is provable in cut-free TN $[l]$.

\section{Time-as-resources correspondence}

In this section, a resource-indexed non-commutative logic $\mathrm{RN}[l]$, which is regarded as a non-commutative version of the resource-indexed linear logic $\mathrm{RL}[l]$ proposed in [10], is introduced, and the correspondence between $\mathrm{RN}[l]$ and $\mathrm{TN}[l]$ is shown.

The proposed logic RN $[l]$ is obtained from FL by adding modified $l$ bounded exponential operators $!_{l}$ and $?_{l}$ and generalizing initial sequents and inference rules by putting explicit resource indexes. The operator $!_{l}$ is characterized by the following inference rules: for a fixed positive integer $l$,

$$
\frac{\Gamma, \overbrace{\alpha * \cdots * \alpha}^{1 \leq n \leq l}, \Delta \Rightarrow \gamma}{\Gamma, !_{l} \alpha, \Delta \Rightarrow \gamma} \quad \frac{\Gamma \Rightarrow \alpha \quad \Gamma \Rightarrow \alpha * \alpha \quad \ldots \quad \Gamma \Rightarrow \overbrace{\alpha * \cdots * \alpha}^{l+1}}{\Gamma \Rightarrow !_{l} \alpha}
$$

which correspond to the Hilbert-style axiom scheme:

$$
!_{l} \alpha \leftrightarrow \alpha \wedge(\alpha * \alpha) \wedge(\alpha * \alpha * \alpha) \wedge \cdots \wedge(\overbrace{\alpha * \cdots * \alpha}^{l+1}) .
$$

Since the intended meaning of the formula of the form $\overbrace{\alpha * \cdots * \alpha}^{i}$ is:

- "The resource $\alpha$ is usable just in the number $i$, but only once",

the intended meaning of the formula of the form $!_{l} \alpha$ is:

- "The resource $\alpha$ is usable in any finite positive number less than $l$, but only once (i.e., it is consumed after use)".

In this section, the same notations and conventions as in the previous sections are also adopted. The language of the logics which are introduced in this section is obtained from that of $\mathrm{TN}[l]$ by deleting $\mathrm{X}, \mathrm{G}$ and $\mathrm{F}$ and adding 
$!_{l}$ (l-bounded exponential operator or $l$-bounded "of course" operator) and $?_{l}$ (l-bounded "why not" operator). For any $\sharp \in\left\{!_{l}, ?_{l}\right\}$, an expression $\sharp \Gamma$ is used to denote the sequence $\langle\sharp \gamma \mid \gamma \in \Gamma\rangle$. An expression $\alpha^{i}$ for any $i \in \omega$ is used to denote $\overbrace{\alpha * \alpha * \cdots * \alpha}^{i+1}$. In this expression, the superscript ${ }^{i}$ of $\alpha$ is called the resource index of $\alpha$. The intended meaning of $\alpha^{i}$ is thus "The resource $\alpha$ is usable just in the number $i+1$, but only once".

The logic $\mathrm{RN}[l]$ is introduced below.

Definition $4.22(\mathrm{RN}[l])$. An expression $\alpha^{i}$ with $i \in \omega$ is inductively defined by $\left(\alpha^{0}:=\alpha\right)$ and $\left(\alpha^{i+1}:=\alpha^{i} * \alpha\right)$. Let $l$ be a fixed positive integer (called a resource bound).

The initial sequents of $\mathrm{RN}[l]$ are of the form: for any atomic formula $p$,

$$
p^{i} \Rightarrow p^{i} \quad \Rightarrow \mathbf{1}^{i} \quad \Gamma, \perp^{i}, \Delta \Rightarrow \gamma \quad \Gamma \Rightarrow \top^{i} .
$$

The cut rule of $\mathrm{RN}[l]$ is of the form:

$$
\frac{\Gamma \Rightarrow \alpha \quad \Sigma, \alpha, \Delta \Rightarrow \gamma}{\Sigma, \Gamma, \Delta \Rightarrow \gamma} \text { (cut). }
$$

The logical inference rules of $\mathrm{RN}[l]$ are of the form: for any $k \in \omega_{l}$ and any positive integer $m$,

$$
\begin{aligned}
& \frac{\Gamma, \Delta \Rightarrow \gamma}{\Gamma, \mathbf{1}^{i}, \Delta \Rightarrow \gamma}\left(\mathbf{1} \mathrm{we}^{r}\right) \\
& \frac{\Gamma \Rightarrow \alpha^{i} \quad \Sigma, \beta^{i}, \Delta \Rightarrow \gamma}{\Sigma,(\alpha \rightarrow \beta)^{i}, \Gamma, \Delta \Rightarrow \gamma}\left(\rightarrow \operatorname{left}^{r}\right) \quad \frac{\Gamma, \alpha^{i} \Rightarrow \beta^{i}}{\Gamma \Rightarrow(\alpha \rightarrow \beta)^{i}}\left(\rightarrow \text { right }^{r}\right) \\
& \frac{\Gamma \Rightarrow \alpha^{i} \quad \Sigma, \beta^{i}, \Delta \Rightarrow \gamma}{\Sigma, \Gamma,(\alpha \leftarrow \beta)^{i}, \Delta \Rightarrow \gamma}\left(\leftarrow \mathrm{left}^{r}\right) \quad \frac{\alpha^{i}, \Gamma \Rightarrow \beta^{i}}{\Gamma \Rightarrow(\alpha \leftarrow \beta)^{i}}\left(\leftarrow \operatorname{right}^{r}\right) \\
& \frac{\Gamma, \alpha^{i}, \Delta \Rightarrow \gamma}{\Gamma,(\alpha \wedge \beta)^{i}, \Delta \Rightarrow \gamma}\left(\wedge \operatorname{left}^{r}\right) \quad \frac{\Gamma, \beta^{i}, \Delta \Rightarrow \gamma}{\Gamma,(\alpha \wedge \beta)^{i}, \Delta \Rightarrow \gamma}\left(\wedge \operatorname{left}^{r}\right) \\
& \frac{\Gamma \Rightarrow \alpha^{i} \quad \Gamma \Rightarrow \beta^{i}}{\Gamma \Rightarrow(\alpha \wedge \beta)^{i}}\left(\wedge \text { right }^{r}\right) \quad \frac{\Gamma, \alpha^{i}, \Delta \Rightarrow \gamma \quad \Gamma, \beta^{i}, \Delta \Rightarrow \gamma}{\Gamma,(\alpha \vee \beta)^{i}, \Delta \Rightarrow \gamma}\left(\text { Vleft }^{r}\right) \\
& \frac{\Gamma \Rightarrow \alpha^{i}}{\Gamma \Rightarrow(\alpha \vee \beta)^{i}}\left(\operatorname{Vright}^{r}\right) \quad \frac{\Gamma \Rightarrow \beta^{i}}{\Gamma \Rightarrow(\alpha \vee \beta)^{i}}\left(\operatorname{Vright}^{r}\right) \\
& \frac{\Gamma, \alpha^{i}, \beta^{i}, \Delta \Rightarrow \gamma}{\Gamma,(\alpha * \beta)^{i}, \Delta \Rightarrow \gamma}\left(* \operatorname{left}^{r}\right) \quad \frac{\Gamma \Rightarrow \alpha^{i} \quad \Delta \Rightarrow \beta^{i}}{\Gamma, \Delta \Rightarrow(\alpha * \beta)^{i}}\left(\text { right }^{r}\right)
\end{aligned}
$$




$$
\begin{array}{cc}
\frac{\Gamma, \alpha(t)^{i}, \Delta \Rightarrow \gamma}{\Gamma,(\forall x \alpha(x))^{i}, \Delta \Rightarrow \gamma}\left(\forall \text { left }^{r}\right) & \frac{\Gamma \Rightarrow \alpha(a)^{i}}{\Gamma \Rightarrow(\forall x \alpha(x))^{i}}\left(\forall \text { right }^{r}\right) \\
\frac{\Gamma, \alpha(a)^{i}, \Delta \Rightarrow \gamma}{\Gamma,(\exists x \alpha(x))^{i}, \Delta \Rightarrow \gamma}\left(\exists \text { left }^{r}\right) & \frac{\Gamma \Rightarrow \alpha(t)^{i}}{\Gamma \Rightarrow(\exists x \alpha(x))^{i}}\left(\exists \text { right }^{r}\right) \\
\frac{\Gamma, \alpha^{l}, \Delta \Rightarrow \gamma}{\Gamma, \alpha^{l+m}, \Delta \Rightarrow \gamma} \text { (INleft) } & \frac{\Gamma \Rightarrow \alpha}{\Gamma \Rightarrow \alpha^{l+m}} \text { (INright) } \\
\frac{\Gamma, \alpha^{i+k}, \Delta \Rightarrow \gamma}{\Gamma,\left(!_{l} \alpha\right)^{i}, \Delta \Rightarrow \gamma}\left(!_{l} \text { left }\right) & \frac{\left\{\Gamma \Rightarrow \alpha^{i+j}\right\}_{j \in \omega_{l}}}{\Gamma \Rightarrow\left(!_{l} \text { right }\right)} \\
\frac{\left\{\Gamma, \alpha^{i+j}, \Delta\right)^{i}}{\left.\Gamma,\left(?_{l} \alpha\right)^{i}, \Delta \Rightarrow \gamma\right\}_{j \in \omega_{l}}}\left(?_{l} \text { left }\right) & \left.\frac{\Gamma \Rightarrow \alpha^{i+k}}{\Gamma \Rightarrow\left(?_{l} \alpha\right)^{i}} \text { (? } ?_{l} \text { right }\right)
\end{array}
$$

where $a$ in $\left(\forall\right.$ right $\left.^{r}\right)$ and $\left(\exists \mathrm{left}^{r}\right)$ is a free variable which must not occur in the lower sequents of the rules, and $t$ in $\left(\forall \operatorname{left}^{r}\right)$ and $\left(\exists \operatorname{right}^{r}\right)$ is an arbitrary term.

Definition $4.23(\mathrm{RN}[\omega])$. The logic $\mathrm{RN}[\omega]$ is obtained from $\mathrm{RN}[l]$ by deleting $\{$ (INleft), (INright) $\}$ and replacing $\omega_{l}$ by $\omega$. The modified rules obtained from (! left), (! right), (? $?_{l}$ left) and (? $?_{l}$ right) by replacing $\omega_{l}$ by $\omega$ are denoted as (! ${ }_{\omega}$ left $),\left(!_{\omega}\right.$ right $),\left(?_{\omega}\right.$ left $)$ and $\left(?_{\omega}\right.$ right $)$, respectively.

Remark that for any formula $\alpha$, the sequent of the form $\alpha^{i} \Rightarrow \alpha^{i}$ is provable in $\mathrm{RN}[l]$. This can be shown by induction on $\alpha$.

A commutative (linear logic) version RL[l] was introduced in [10]. But, the setting of $\mathrm{RL}[l]$ is somewhat different from $\mathrm{RN}[l]$ : $\mathrm{RL}[l]$ has no $\{$ (INleft), (INright)\}, and has the structural rule of exchange. The correspondence between $\mathrm{RL}[l]$ and the commutative version of linear-time temporal logic was not discussed in [10]. In this section, the correspondence between $\mathrm{RN}[l]$ and $\mathrm{TN}[l]$ is shown.

The logic $R N[\omega]$ is, roughly speaking, regarded as an intuitionistic and non-commutative version of Baratella and Masini's 2-sequent calculus 2S $\omega$ for (classical) linear-time temporal logic [2], where $!_{l}$ and $?_{l}$ are replaced by G and $\mathrm{F}$, respectively. But, strictly speaking, $\mathrm{RN}[\omega]$ is not just the 2 -sequent calculus because in $2 \mathrm{~S} \omega$, the index ${ }^{i}$ of $\alpha^{i}$ is just used as a label, but in $\mathrm{RN}[\omega]$, the index.$^{i}$ of $\alpha^{i}$ is just the abbreviation of the formula $\overbrace{\alpha * \cdots * \alpha}^{i+1}$. Since the treatment of the infinite rules (! ${ }_{\omega}$ right) and (? ${ }_{\omega}$ left) in $\mathrm{RN}[\omega]$ is difficult, we do not know whether $\mathrm{RN}[\omega]$ is decidable or not. This problem is also remained open. We do not discuss more about $\mathrm{RN}[\omega]$. 
Proposition 4.24. Let $m$ and $n$ be distinct fixed positive integers. The logics $\mathrm{RN}[m]$ and $\mathrm{RN}[n]$ are not theorem-equivalent.

Proposition 4.25. The $\left\{!_{l}, ?_{l}\right\}$-free fragment $\mathrm{RN}$ of $\mathrm{RN}[l]$ is strictly stronger than FL.

Proof. For an atomic formula $p$, the sequent $p^{2} \rightarrow p^{2} \Rightarrow(p \rightarrow p)^{2}$ is not provable in $\mathrm{FL}$, but it is provable in $\mathrm{RN}$.

Although $\mathrm{RN}[l]$ and $\mathrm{TN}[l]$ are quite resemble, these logics are different.

Proposition 4.26. There is a formula which is provable in the $\left\{!_{l}, ?_{l}\right\}$-free fragment of $\mathrm{RN}[l]$ but not provable in $\mathrm{TN}[l]$.

Proof. Take the same example as in the proof of Proposition 4.25.

Definition 4.27. We fix a countable non-empty set $\Phi$ of atomic formulas, and define the sets $\Phi_{i}:=\left\{p_{i} \mid p \in \Phi\right\}(1 \leq i \in \omega)$ and $\Phi_{0}:=\Phi$ of atomic formulas. The language $\mathcal{L}_{\mathrm{RN}[l]}$ (or the set of formulas) of $\mathrm{RN}[l]$ is defined by using $\Phi, \mathbf{1}, \top, \perp, \rightarrow, \leftarrow, \wedge, \vee, *, \forall, \exists, !_{l}$ and $?_{l}$. The language $\mathcal{L}_{\mathrm{FL}}$ of FL is defined by using $\bigcup_{i \in \omega} \Phi_{i}, \mathbf{1}, \top, \perp, \rightarrow, \leftarrow, \wedge, \vee, *, \forall$ and $\exists$.

A mapping $g$ from $\mathcal{L}_{\mathrm{RN}[l]}$ to $\mathcal{L}_{\mathrm{FL}}$ is defined by: for any $i \in \omega$ and any positive integer $m$,

1. $g\left(p^{i}\right):=p_{i} \in \Phi_{i}$ for any $p \in \Phi$ (especially, $g(p):=p \in \Phi_{0}$ ),

2. $g\left(\sharp^{i}\right):=\sharp$ where $\sharp \in\{\mathbf{1}, \top, \perp\}$,

3. $g\left((\alpha \circ \beta)^{i}\right):=g\left(\alpha^{i}\right) \circ g\left(\beta^{i}\right)$ where $\circ \in\{\rightarrow, \leftarrow, \wedge, \vee, *\}$,

4. $g\left((Q \alpha(x))^{i}\right):=Q g\left(\alpha(x)^{i}\right)$ where $Q \in\{\forall x, \exists x\}$,

5. $g\left(\alpha^{l+m}\right):=g\left(\alpha^{l}\right)$,

6. $g\left(\left(!_{l} \alpha\right)^{i}\right):=\bigwedge\left\{g\left(\alpha^{i+j}\right) \mid j \in \omega_{l}\right\}$,

7. $g\left(\left(?_{l} \alpha\right)^{i}\right):=\bigvee\left\{g\left(\alpha^{i+j}\right) \mid j \in \omega_{l}\right\}$.

An expression $g(\Gamma)$ denotes the result of replacing every occurrence of a formula $\alpha$ in $\Gamma$ by an occurrence of $g(\alpha)$.

The embedding function $g$ is strongly dependent on the time bound $l$ (i.e., $g$ should be denoted as $g_{l}$ ). But, for the sake of brevity, a simple expression $g$ will be used in the following. 
TheOREM 4.28 (Embedding). Let $\Gamma$ be a sequence of formulas in $\mathcal{L}_{\mathrm{RN}[l]}$, $\gamma$ be a formula in $\mathcal{L}_{\mathrm{RN}[l]}$, and $g$ be the mapping defined in Definition 4.27. Then:

1. $\mathrm{RN}[l] \vdash \Gamma \Rightarrow \gamma$ iff $\mathrm{FL} \vdash g(\Gamma) \Rightarrow g(\gamma)$.

2. $\mathrm{RN}[l]-$ (cut) $\vdash \Gamma \Rightarrow \gamma$ iffFL $-($ cut $) \vdash g(\Gamma) \Rightarrow g(\gamma)$.

Proof. Similar to Theorem 2.7.

Using Theorem 4.28, we obtain the following theorems.

Theorem 4.29 (Cut-elimination). The rule (cut) is admissible in cut-free $\mathrm{RN}[l]$.

Theorem 4.30 (Decidability). RN[l] is decidable.

Definition 4.31. Let $\mathcal{L}_{\mathrm{TN}[l]}$ and $\mathcal{L}_{\mathrm{RN}[l]}$ be the languages defined in definitions 2.6 and 4.27. Then:

1. A mapping $h_{1}$ from $\mathcal{L}_{\mathrm{TN}[l]}$ to $\mathcal{L}_{\mathrm{RN}[l]}$ is defined by $h_{1}\left(\mathrm{X}^{i} \alpha\right):=\alpha^{i}$.

2. A mapping $h_{2}$ from $\mathcal{L}_{\mathrm{RN}[l]}$ to $\mathcal{L}_{\mathrm{TN}[l]}$ is defined by $h_{2}\left(\alpha^{i}\right):=\mathrm{X}^{i} \alpha$.

Note that $h_{2} h_{1}\left(\mathrm{X}^{i} \alpha\right)=\mathrm{X}^{i} \alpha$ and $h_{1} h_{2}\left(\alpha^{i}\right)=\alpha^{i}$.

Although, as mentioned before (Proposition 4.26), TN $[l]$ and $\mathrm{RN}[l]$ are different logics, the following correspondence theorem, which means "time is regarded as a resource", can straightforwardly be obtained. This theorem is regarded as an intuitionistic, non-commutative and bounded version of the correspondence theorem of $\mathrm{LT}_{\omega}$ and $2 \mathrm{~S} \omega$, which was presented in [8].

TheOrem 4.32 (Correspondence between TN[l] and RN[l]). Let $h_{1}$ and $h_{2}$ be the mappings defined in Definition 4.31. Let $\Gamma$ be a sequence of formulas in $\mathcal{L}_{\mathrm{RN}[l]}$ and $\gamma$ be a formula in $\mathcal{L}_{\mathrm{RN}[l]}$. Let $\Gamma^{\prime}$ be a sequence of formulas in $\mathcal{L}_{\mathrm{TN}[l]}$ and $\gamma^{\prime}$ be a formula in $\mathcal{L}_{\mathrm{TN}[l]}$. Then:

1. $\mathrm{TN}[l] \vdash \Gamma \Rightarrow \gamma$ iff $\mathrm{RN}[l] \vdash h_{1}(\Gamma) \Rightarrow h_{1}(\gamma)$.

2. $\mathrm{TN}[l]-$ (cut) $\vdash \Gamma \Rightarrow \gamma$ iff $\mathrm{RN}[l]-($ cut $) \vdash h_{1}(\Gamma) \Rightarrow h_{1}(\gamma)$.

3. $\mathrm{RN}[l] \vdash \Gamma \Rightarrow \gamma$ iff $\mathrm{TN}[l] \vdash h_{2}(\Gamma) \Rightarrow h_{2}(\gamma)$.

4. $\mathrm{RN}[l]-$ (cut) $\vdash \Gamma \Rightarrow \gamma$ iff $\mathrm{TN}[l]-($ cut $) \vdash h_{2}(\Gamma) \Rightarrow h_{2}(\gamma)$. 
Using Theorem 4.32, we can obtain alternative proofs of the cut-elimination theorems for $\mathrm{TN}[l]$ and $\mathrm{RN}[l]$. We now show only the alternative proof of the cut-elimination theorem for $\mathrm{TN}[l]$. Suppose $\mathrm{TN}[l] \vdash \Gamma \Rightarrow \gamma$. Then, we obtain $\mathrm{RN}[l] \vdash h_{1}(\Gamma) \Rightarrow h_{1}(\gamma)$ by Theorem $4.32(1)$. We then obtain $\mathrm{RN}[l]-$ (cut) $\vdash h_{1}(\Gamma) \Rightarrow h_{1}(\gamma)$ by Theorem 4.29. Thus, we get TN $[l]$ - (cut) $\vdash h_{2} h_{1}(\Gamma) \Rightarrow h_{2} h_{1}(\gamma)$ by Theorem 4.32(4). Since $h_{2} h_{1}(\Gamma)-=\Gamma$ and $h_{2} h_{1}(\gamma)=\gamma$, we have the required fact $\mathrm{TN}[l]-$ (cut) $\vdash \Gamma \Rightarrow \gamma$.

\section{Concluding remarks}

In this paper, a first-order temporal non-commutative logic $\mathrm{TN}[l]$, which has no structural rules and has some $l$-bounded linear-time temporal operators, was introduced, and the decidability, cut-elimination and completeness (w.r.t. phase semantics) theorems for $\mathrm{TN}[l]$ were shown using the embedding theorem of TN $[l]$ into the full Lambek logic FL. The logic TN $[l]$ can represent not only time-dependent, resource-sensitive, ordered, but also hierarchical reasoning. An advantage of $\mathrm{TN}[l]$ is regarded as its decidability. The correspondence theorem between $\mathrm{TN}[l]$ and a resource-indexed non-commutative logic $\mathrm{RN}[l]$ was shown. This theorem was intended to represent the "timeas-resources" interpretation.

In the following, some remarks on substructural extensions are given. We can obtain some substructural extensions of $\mathrm{TN}[l]$ and $\mathrm{RN}[l]$ by adding some structural rules. We can also obtain similar results on the embedding, cut-elimination, completeness and correspondence results for these extensions. Although we cannot obtain the decidability results for the extensions with the contraction rule (these logics are indeed undecidable), we can obtain the decidability results for some logics without the contraction rule. For example, the linear logic version TL $[l]$ of $\mathrm{TN}[l]$ is obtained from $\mathrm{TN}[l]$ by adding the exchange rule. Then, the embedding, cut-elimination, decidability, completeness and correspondence theorems for TL $[l]$ can be obtained in a similar way.

Acknowledgments. This research was supported by the Alexander von Humboldt Foundation and by the Japanese Ministry of Education, Culture, Sports, Science and Technology, Grant-in-Aid for Young Scientists (B) 20700015 . 


\section{References}

[1] Abrusci, V. M., "Non-commutative intuitionistic linear logic", Ztschr. f. Math. Logik 36 (1990): 297-318.

[2] Baratella, S., and A. Masini, "An approach to infinitary temporal proof theory", Archive for Mathematical Logic 43, 8 (2004): 965-990.

[3] Biere, A., A. Cimatti, E. M. Clarke, O. Strichman and Y. Zhu, "Bounded model checking", Advances in Computers 58 (2003): 118-149.

[4] Cerrito, S., M. C. Mayer and S. Prand, "First order linear temporal logic over finite time structures", pp. 62-76 in: Proceedings of the 6th International Conference on Logic for Programming and Automated Reasoning (LPAR'99), Lecture Notes in Computer Science 1705, Springer-Verlag, Berlin, 1999.

[5] Girard, J.-Y., "Linear logic", Theoretical Computer Science 50 (1987): 1-102.

[6] Hodkinson, I., F. Wolter and M. Zakharyaschev, "Decidable fragments of firstorder temporal logics", Annals of Pure and Applied Logic 106 (2000): 85-134.

[7] Kamide, N., "Linear and affine logics with temporal, spatial and epistemic operators", Theoretical Computer Science 353, 1-3 (2006): 165-207.

[8] Kamide, N., "An equivalence between sequent calculi for linear-time temporal logic", Bulletin of the Section of the Logic 35, 4 (2006): 187-194.

[9] Kamide, N., "A uniform proof-theoretic foundation for abstract paraconsistent logic programming", Journal of Functional and Logic Programming, 2007.

[10] Kamide, N., "Linear exponentials as resource operators: A decidable firstorder linear logic with bounded exponentials", pp. 245-257 in: Proceedings of the 11th European Conference on Logics in Artificial Intelligence (JELIA2008), Lecture Notes in Artificial Intelligence 5293, 2008.

[11] Kamide, N., "Embedding linear-time temporal logic into infinitary logic: Application to cut-elimination for multi-agent infinitary epistemic linear-time temporal logic", pp. 57-76 in Proceedings of the 9th International Workshop on Computational Logic in Multi-agent Systems (CLIMA-9), Lecture Notes in Artificial Intelligence 5405, 2009.

[12] Kawai, H., "Sequential calculus for a first order infinitary temporal logic", Zeitschrift für Mathematische Logik und Grundlagen der Mathematik 33 (1987): 423-432.

[13] Komori, Y., "Predicate logics without the structure rules", Studia Logica 45 (1986): 393-404.

[14] Kröger, F., "LAR: a logic of algorithmic reasoning", Acta Informatica 8 (1977): 243-266. 
[15] Lambek, J., "The mathematics of sentence structure", The American Mathematical Monthly 65 (1958): 154-170.

[16] Okada, M., "A uniform semantic proof for cut-elimination and completeness of various first and higher order logics", Theoretical Computer Science 281 (2002): 471-498.

[17] Pym, D. J., P. W. O'Hearn and H. Yang, "Possible worlds and resources: The semantics of BI", Theoretical Computer Science 315, 1 (2004): 257-305.

[18] Russell, S., and P. Norvig, Artificial intelligence: A modern approach (Second edition), Pearson Education, Inc., 2003.

NORIHIRO KAMIDE

Waseda Institute for Advanced Study

Waseda University

1-6-1 Nishi Waseda, Shinjuku-ku

Tokyo 169-8050, Japan

logician-kamide@aoni. waseda.jp 